ACCEPTED MANUSCRIPT

\title{
A solvable model for the basic properties of a simple magnetized plasma torus
}

To cite this article before publication: Ashild Fredriksen et al 2018 Plasma Phys. Control. Fusion in press https://doi.org/10.1088/1361$\underline{6587 / \text { aacd2a }}$

\author{
Manuscript version: Accepted Manuscript \\ Accepted Manuscript is "the version of the article accepted for publication including all changes made as a result of the peer review process, \\ and which may also include the addition to the article by IOP Publishing of a header, an article ID, a cover sheet and/or an 'Accepted \\ Manuscript' watermark, but excluding any other editing, typesetting or other changes made by IOP Publishing and/or its licensors" \\ This Accepted Manuscript is @ 2018 IOP Publishing Ltd.
}

During the embargo period (the 12 month period from the publication of the Version of Record of this article), the Accepted Manuscript is fully protected by copyright and cannot be reused or reposted elsewhere.

As the Version of Record of this article is going to be / has been published on a subscription basis, this Accepted Manuscript is available for reuse under a CC BY-NC-ND 3.0 licence after the 12 month embargo period.

After the embargo period, everyone is permitted to use copy and redistribute this article for non-commercial purposes only, provided that they adhere to all the terms of the licence https://creativecommons.org/licences/by-nc-nd/3.0

Although reasonable endeavours have been taken to obtain all necessary permissions from third parties to include their copyrighted content within this article, their full citation and copyright line may not be present in this Accepted Manuscript version. Before using any content from this article, please refer to the Version of Record on IOPscience once published for full citation and copyright details, as permissions will likely be required. All third party content is fully copyright protected, unless specifically stated otherwise in the figure caption in the Version of Record.

View the article online for updates and enhancements. 


\title{
A solvable model for the basic properties of a simple magnetized plasma torus
}

\author{
Å Fredriksen ${ }^{1} \mathrm{H} \mathrm{L}$ Pécseli ${ }^{2} \mathrm{~J} \mathrm{~K}_{\text {Trulsen }}{ }^{3}$ \\ 1 Arctic University of Norway, Department of Physics and Technology, N-9037 \\ Troms $\varnothing$, Norway \\ E-mail: hans.pecseli@fys.uio.no \\ ${ }^{2}$ University of Oslo, Department of Physics, P.O. Boks 1048 Blindern, N-0316 Oslo, \\ Norway \\ 3 University of Oslo, Institute of Theoretical Astrophysics, P.O. Boks 1029 Blindern, \\ N-0315 Oslo, Norway
}

\begin{abstract}
A simple magnetized plasma torus is modeled by using a "top hat" density variation. The benefit of this simplification is an exactly solvable model, allowing for a few additions, such as an externally maintained parabolic steady state potential variation imposed on the plasma. The combined effects of the resulting plasma rotation and the plasma polarization due to magnetic field gradient particle drifts can be described. The stabilizing and confining effects of plasma rotation are explicitly demonstrated. In a special high plasma density limit the results are confirmed by a more general model, indicating that the results of the top-hat model can be used with confidence in more general cases. A small vertical magnetic field component can be included for a plasma with neutral collisions and its influence on the electron dynamics studied. The effects of ion - neutral collisions are also included. The rotation and polarization of the plasma has different effects on the time variations of the plasma density and potential. As a reference we use data from the Blaamann device at the University of Troms $\varnothing$ obtained by a movable multi-probe, measuring variations in density, floating potential and an electric field component. The fluctuations in the plasma are characterized by auto-correlations and by cross-correlations between the signal from a fixed reference probe and data. The model accounts adequately for the phase variations of the signals for varying spatial multi-probe positions.
\end{abstract}

PACS numbers: 52.25.Fi, 52.35.Ra

Magnetized plasmas, Plasma equilibrium, Correlation measurements: Article preparation, IOP journals

Submitted to: Plasma Phys. Control. Fusion 


\section{Basic features of magnetized plasma torii}

\section{Introduction}

It is well known that a simple magnetized plasma torus has no equilibrium, and a perturbation analysis in the classical sense is thus meaningless; there is no equilibrium solution to perturb. A key problem in previous studies of the confinement of hot fusion plasmas addressed modifications of such simple torii in order to obtain stable or semistable equilibria. It was found, however, that in spite of their basic shortcomings, continuously generated cold plasmas in simple torii had interesting properties and several such devices $[1,2,3,4,5]$ have been built and operated. The plasma losses are here continuously compensated by a plasma discharge, for instance [6, 7]. There are differences in the details of the set-up, for instance concerning the positioning of discharge filaments, etc. Unless an RF-discharge is used, the discharge bias often has a value larger than needed for ionizing the background filling gas. In a discharge plasma as in the magnetized Blaamann device this large negative bias gave a significant enhancement of the plasma density. The analysis presented in the following indicates that plasma rotation in a steady state parabolic potential well has a stabilizing effect. We present analytical results for a scaling law explaining this result.

Some experiments found an advantage in imposing a small vertical magnetic field component, giving more stable plasma conditions. Although many devices are operated, there is thus seemingly no consensus on details in the operations and the role of the externally imposed parameters. Due to the basic toroidal magnetic field, the perturbations in plasma density and the fluctuating electrostatic field are not directly related, as would be the case when the electrons can flow freely to achieve a local isothermal Boltzmann equilibrium. The phase variations of the fluctuations in plasma density and the plasma potential are consequently different.

Analytical models for the plasma discharge have been suggested [1] to account for the plasma production, the transport of plasma as it is confined by the toroidal magnetic field and influenced by a steady state as well as a time varying electric field, and the ultimate plasma losses to the walls of the confining metal vessel. The present study considers elements of this process, namely the polarization of the magnetized plasma column and the resulting transport. The relative phase of fluctuations in density and potential is also accounted for. The advantage of this approach is that it allows a simple description in the form of an initial value problem. The shortcoming is the missing account of the continuous plasma production. We will find nonetheless that our model gives a satisfactory description of the polarization and the basic parameter dependence of the dynamics of the plasma column.

For reference we summarize a set of data from fluctuation measurements obtained in the Blaamann device at the University of Troms $\varnothing$ [1]. The plasma was maintained by a discharge from a vertical, hot, electron emitting filament, placed centrally in the cross-section of the vessel. The plasma column was terminated radially by a grounded limiter extending $2.5 \mathrm{~cm}$ into the plasma from the wall of the toroidal vessel. The ratio of the minor to major radius of the toroidal stainless steel chamber was $r_{0} / R_{0} \approx 0.2$. The 


\section{Basic features of magnetized plasma torii}

limiter reduces the effective minor radius of the plasma column. The dominant magnetic field had a toroidal symmetry with the option of adding a small vertical magnetic field component, for instance for compensating the Earth's magnetic field which is nearly vertical in Troms $\varnothing$. A summary of the basic steady state plasma parameters is given in Appendix A. Fluctuations in the local plasma density and floating potentials are analyzed by high-pass filtering the signal from the probes at $300 \mathrm{~Hz}$. The fluctuation measurements are summarized in terms of auto-correlation functions for the signals as well as by cross-correlations with the potential obtained by a fixed reference probe, see Sections 2 and 3. The data are included to illustrate the relative phase variations for varying spatial positions for the fluctuations in electrostatic potential and plasma density.

We suggest, in Section 4, a simple analytical model that follows a plasma crosssection as it moves by the $\mathbf{E} \times \mathbf{B} / B^{2}$ drifts. The electric field is given in part by an imposed nearly parabolic stationary potential well, and in part caused by the polarization of the plasma. At first, the general model is presented, and its features then discussed by a step-by-step illustration by including first the parabolic potential in addition to the basic polarization drift (with supporting material placed in Appendix B), and then the short circuiting effect of the small vertical magnetic field component. Also ion collision effects are considered. For most realistic cases we find that the plasma will eventually be lost to the walls due to these plasma drifts, although a significant stabilizing effect is found by the externally imposed parabolic potential. A steady state model assumes that plasma cross-sections are continuously maintained to compensate for the losses $[6,7]$.

Our model also serves to illustrate some inherent problems associated with the usual AC coupling of the circuit detecting temporal potential variations. The contribution to the anomalous plasma flux due to steady-state and low frequency plasma variations with $\omega \ll \Omega_{c i}$ can be written as $(\bar{n}(\mathbf{r})+\widetilde{n}(\mathbf{r}, t))(\overline{\mathbf{E}}(\mathbf{r})+\widetilde{\mathbf{E}}(\mathbf{r}, t)) \times \mathbf{B} / B^{2}$, with overlines denoting time averages. In our case we have $\overline{\mathbf{E}}$ including an externally imposed radial electric field $\mathbf{E}_{0}$ giving a rotation of the plasma, and an electric field component caused by polarization of the plasma column. Due to the AC couplings of the potential probes the contributions to the average losses induced by the part containing $\overline{\mathbf{E}}$ can not be determined. These can be dominant in many cases.

\section{Probes for data acquisition}

Temporal variations are analyzed by correlations based on data obtained by a fixed reference probe detecting potential variations and a movable multi-probe, later referred to as the signal probe, see Fig. 1 . The circle at a position $(x, y)=(0,5) \mathrm{cm}$ indicates the position of the fixed reference probe. The multi-probe contains two probes, "CH1" and "CH2", detecting floating potentials. Their difference potential divided by the probe separation gives an estimate for the vertical $y$-component, $E_{y}(t)$, of the fluctuating electric field. The probe labeled $\tilde{n}$ measures density variations. The probes are 


\section{Basic features of magnetized plasma torii}

cylindrical, with an exposed length $5 \mathrm{~mm}$ and diameter $0.25 \mathrm{~mm}$. The separation of the centers of the two outermost probes is $7 \mathrm{~mm}$. The probes detecting potential fluctuations were AC coupled, while the probe for density was DC coupled. The probe connections were ceramically insulated, and placed in a grounded $10 \mathrm{~mm}$ diameter stainless steel tube for support.

Filled circles along the $x$-axis in Fig. 1 indicate positions where data were sampled by the movable signal probe. The position $(x, y)=(0,0)$ is placed at the center of the circular cross-section of the vessel. As it turns out, the local minimum of the potential well is approximately at a position $(x, y)=(0,-2.5) \mathrm{cm}$.

Potential measurements give the floating potential $\phi_{f}$ of the probe, where an approximate relation to the plasma potential $\phi_{p}$ is found as

$$
\phi_{p}-\phi_{f} \approx \frac{T_{e}}{e} \log \left(\sqrt{\frac{T_{e} M}{T_{i} m}}\right),
$$

giving the relation between variations in parameters

$$
\Delta \phi_{p}-\Delta \phi_{f} \approx \frac{\Delta T_{e}}{2 e}\left(1+\log \left(\frac{T_{e 0} M}{T_{i} m}\right)\right)
$$

for a plasma with Maxwellian velocity distributions [8]. The potential difference $\phi_{p}-\phi_{f}$ varies with position since the electron temperature depends on position, see Fig. A1. We prefer to show the raw data for floating potential fluctuations, where the corrections due to $T_{e}=T_{e}(\mathbf{r})$ that relate the measurements to the plasma potential can be estimated by (1). The ion temperature $T_{i}$ can be assumed constant for a discharge plasma like that in Blaamann. Within the range of positions for the movable multi-probe (see Fig. 1) we have the electron temperature to vary approximately with a factor of 2 , if we ignore the noisy central part containing the discharge filament. The change in the logarithm in (1) will be small. The floating potential measurements will be used for obtaining an electric field component through a potential difference between the two signals from "CH1" and "CH2". It is implicitly assumed that the two probes are located at the same DC equi-potential curve at all times. A correction due to the spatial electron temperature variation will be negligible here.

The density measurements are affected by the spatial variation of $T_{e}$. We use the electron saturation current where the electron thermal velocity enters. In case the ion saturation is used, the electron temperature enters through the sound speed in the Bohm condition. In either case the effect will vary as $\sim \sqrt{T_{e}}$, which here introduces a factor of $\sqrt{2}$, at most.

The plasma discharge changed slightly when the grounded multi-probe support (or probe shaft) crosses the magnetic flux tube that contains the hot discharge filament. We noted this by a change in the basic frequency detected by the movable as well as the fixed probe. The root mean square (RMS) value of the reference probe signal remains almost constant, only the frequency changes. The basic frequency $\Omega_{0}$ is given by the rotation of the plasma column, as induced by a steady radial electric field $E_{0}$. The corresponding steady potential variation changes slightly due to the multi-probe motion. In effect, 
1

2

3

4

5

6

7

8

\section{Basic features of magnetized plasma torii}

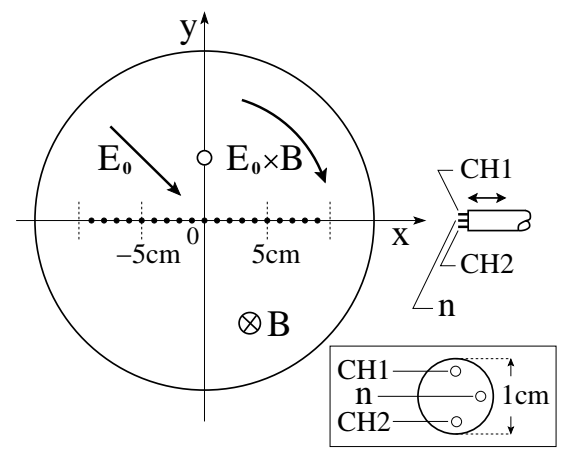

Figure 1. Schematic illustration of the probe set-up for correlation measurements. The fixed reference probe is marked by an open circle. The insert in the lower right corner shows a front view of the movable probe.

the data thus contain results from two sets of experimental conditions. Data from the noisy region near the discharge filament are not used, and are here masked by a white zone. This removes data from two probe positions near the plasma center and the interpolating spatial interval to the adjacent two probe positions.

In Fig. 2 we show the root mean square (RMS) variations of the various quantities detected by the movable probe. Together with the normalized auto-correlation functions this information accounts for the lowest order statistical information of the signals.

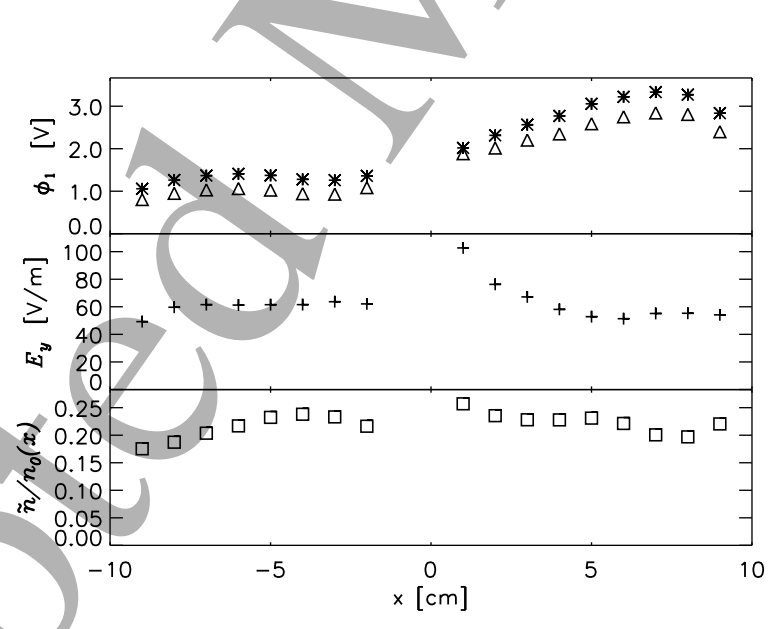

Figure 2. Variation with $x$-position of the RMS-variations of floating potential $\phi$ for channel CH1 and $\mathrm{CH} 2$ with symbols $*$ and $\Delta$, the $y$-component of the electric field, and the local relative density $\widetilde{n} / \bar{n}$. The noisy region in the vicinity of the discharge filament at $x \approx 0$ is omitted in all cases. Concerning the floating potential measurements we recall the expression (1) for the relation to the plasma potential.

We found a slight difference between the root-mean-square (RMS) value of the potential fluctuations in the signal from the two probes, "CH1" and "CH2". This difference in RMS value varied with position, being $\sim 15 \%$ for $x>0$ and slightly larger for $x<0$, i.e. $\sim 25 \%$. The observed difference in the detected fluctuation level for $x<0$ and $x>0$ is most likely caused by probe shaft disturbance mentioned before. We compensated for this difference. Previous studies with this set-up $[9,10]$ did not use 


\section{Basic features of magnetized plasma torii}

this compensation. A plausible explanation for the difference in the detected fluctuation levels between CH1 and CH2 is found in Section 5.

\section{Experimentally obtained correlations}

Some of the features of the model can be analyzed by studying fluctuations, in the toroidal plasma. A data-set is obtained or this purpose. Using these data, crosscorrelations between the fluctuations in floating potential $\phi_{r}(t)$ detected by the fixed reference probe and signals from the moving probe are computed. We find for instance $R_{r, n_{j}}(\tau)=\left\langle\phi_{r}(t) n_{j}(t+\tau)\right\rangle$, and similarly for the other correlations, with $j \in\{-9,-8, \ldots, 0, \ldots, 8,9\}$ indicating the position number,measuring the $x$-coordinate of the movable probe. The correlation results are robust: they were tested by using a simple method removing the average in each sample, and more detailed analysis where the data were high-pass filtered first by Fourier transform at $300 \mathrm{~Hz}$ as an alternative to removing trends [11].

\subsection{Floating potential and electric field measurements}

The basic fluctuation characteristics are illustrated by the normalized auto-correlation of the signals. In Fig. 3 we show $\left\langle\phi_{j}(t) \phi_{j}(t+\tau)\right\rangle /\left\langle\phi_{j}^{2}\right\rangle$ for varying $j$. At $x>0$ we readily note a dominant frequency $\Omega_{0} \approx 57 \times 10^{3} \mathrm{~s}^{-1}$ with period $0.11 \times 10^{-3} \mathrm{~s}$. A slight change is detected at positions $x<0$ for reasons already mentioned. The auto-correlation for the electric field signal $E_{y j}(t)$ is shown in Fig. 4. The auto-correlation has a significant contribution for small time delays $\tau \ll 2 \pi / \Omega_{0}$. The large time-scale contribution to the correlation is due to the bulk plasma rotation in the nearly parabolic potential well, while the fluctuations with short temporal correlations are due to turbulent motions excited in the plasma. The auto-correlations have a dominant peak at small $\tau$, relative to what is found for the potential. This indicates the presence of short-time, small-scale variations in addition to what is associated with the bulk plasma motions giving the distinct spectral peak.

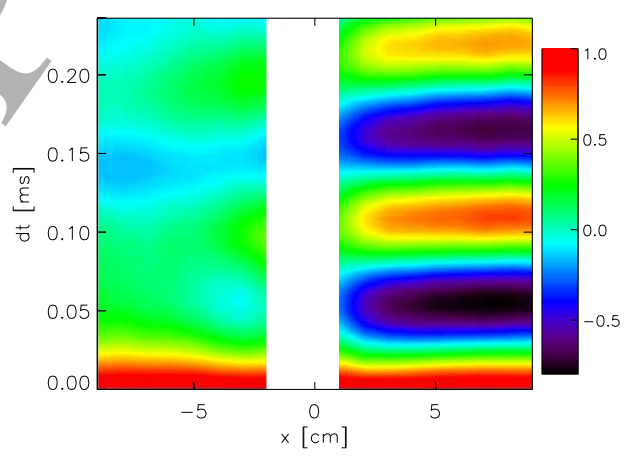

Figure 3. Normalized auto-correlation for the potential fluctuations shown as a function of time separation $\tau$ for varying positions. 


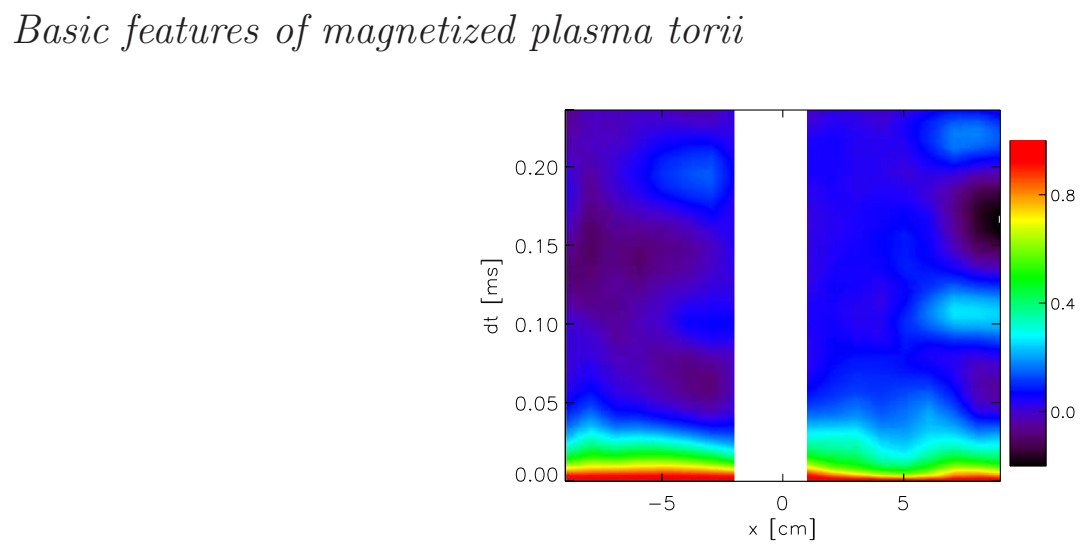

Basic features of magnetized plasma torii

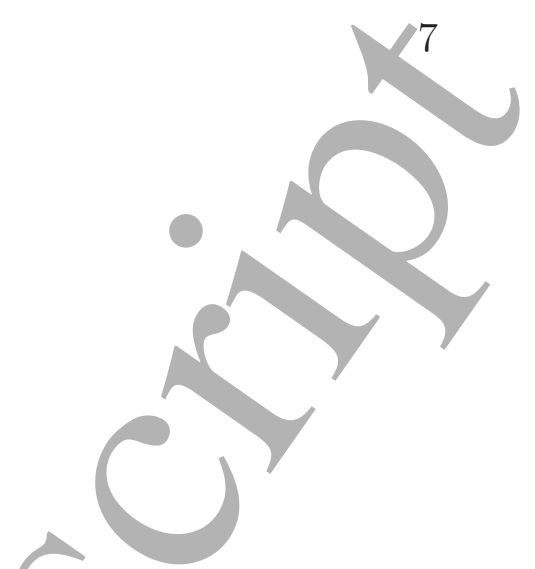

Figure 4. Normalized auto-correlation for the fluctuations in $E_{y} / B$ as a function of time separation $\tau$ for varying positions.

In Fig. 5 we show the cross-correlation between the reference probe signal and the floating potential measured by the signal probe. We find that the fluctuating potential signals are to a good approximation in phase along the entire $x$-axis, in agreement with the suggested model where the polarization of the plasma column is close to vertical at all times, i.e. along the $y$-axis. The normalized correlation is significant: in the range $\{-0.8: 0.5\}$, see color bar to the right of the figure. The correlation is largest for large positive $x$. We note that it takes approximately $0.03 \mathrm{~ms}$ to reach maximum correlation. We take this as an indication of the time it takes a perturbation to propagate the distance of $5 \mathrm{~cm}$ from the reference probe to the $y=0$ axis, corresponding to a downward vertical velocity component of approximately $1.5 \times 10^{3} \pm 10^{2} \mathrm{~m} \mathrm{~s}^{-1}$. We find the most noticeable feature of Fig. 5 to be that at any given time the potential seems to be nearly in phase for all $x$-positions in spite of the plasma rotation. Closer inspection of the phase angle variation shows a small tilt of $5^{\circ}-10^{\circ}$ in the anti-clockwise direction.

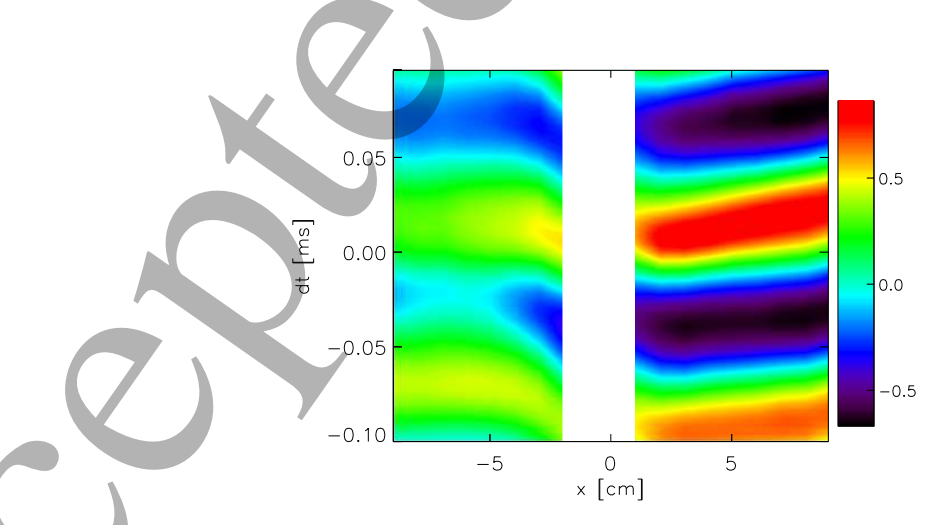

Figure 5. Cross-correlation between the floating potential from the fixed probe (see Fig. 1) and the floating potential detected by the moving probe $\mathrm{CH} 1$.

If the plasma column was displaced by a steady velocity $U$ in the vertical direction, we would expect that the vertical electric field component could be determined by a time derivative of Fig. 5 , assuming $\partial / \partial t \approx-U \partial / \partial y$, where $U$ will vary with radial position, in general. This suggestion can be tested in the experiment since we have two 


\section{Basic features of magnetized plasma torii}

probes available. In particular, for large radial positions (large $x$ values), i.e. at the low magnetic field side, we find this approximation to be reasonably well satisfied. In Fig. 6 we show for completeness also the cross-correlation of $E_{y} / B$ corresponding to Fig. 5. A small phase difference between the variations at $x>0$ and $x<0$ is caused by the probe shaft disturbance mentioned before.

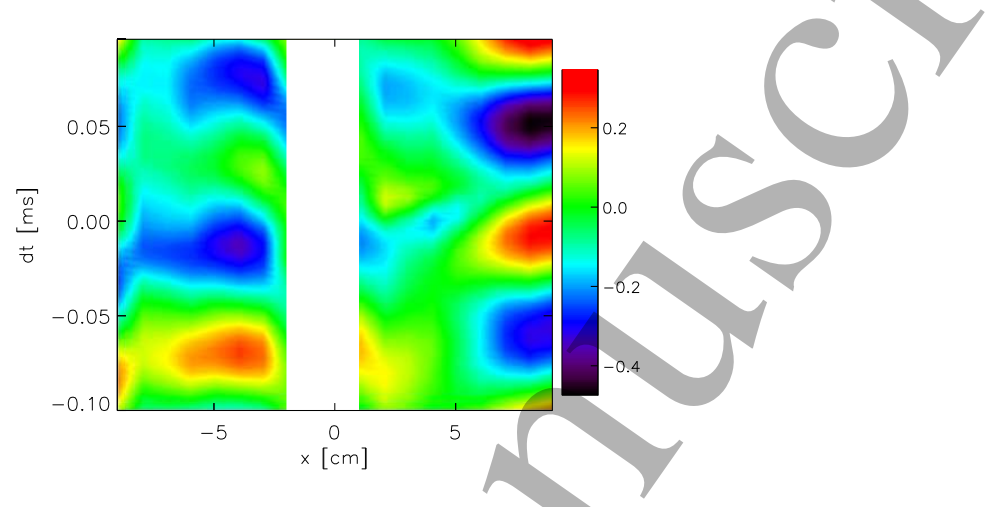

Figure 6. Normalized cross-correlation for the fluctuations in $E_{y}$ and the reference potential signal as a function of time separation $\tau$ for varying positions.

\subsection{Plasma density measurements}

Fluctuations in plasma density, $\widetilde{n}(\mathbf{r}, t)=n(\mathbf{r}, t)-\bar{n}(\mathbf{r})$, are detected by the electron saturation current to the probe marked $\tilde{n}$ in Fig. 1 . In Fig. 7 we show $\left\langle\widetilde{n}_{j}(t) \widetilde{n}_{j}(t+\right.$ $\tau)\rangle /\left\langle\widetilde{n}_{j}^{2}\right\rangle$ for varying $j$. The auto-correlation of the plasma density has the same basic temporal variation as those for the potential, but we note some differences in the numerical magnitudes of the correlation coefficients.

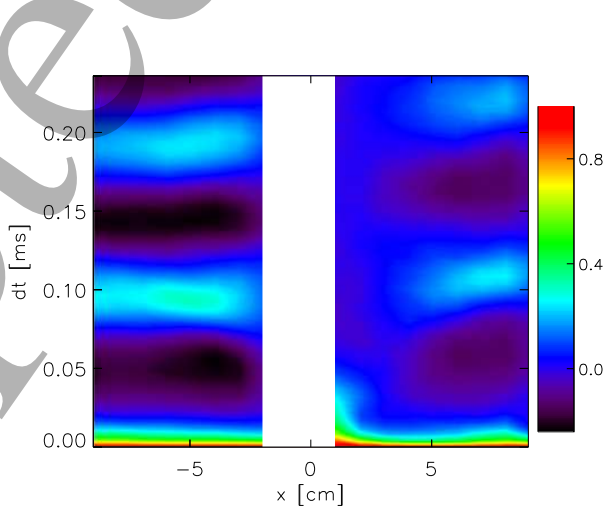

Figure 7. Normalized auto-correlation for the density fluctuations shown as a function of time separation $\tau$ for varying positions.

In Fig. 8 we show cross-correlations between potential fluctuations detected by the reference probe and variations in the plasma density measured by the multi-probe. The normalized correlation is here in the range $\{-0.6: 0.4\}$. Near the position of the discharge filament at $x \approx 0$ we find spurious correlations originating from the discharge 


\section{Basic features of magnetized plasma torii}

noise. The conspicuous feature, as compared to the potential correlations in Fig. 5, is the pronounced time delays in the cross-correlation for large $|x|$. Note that the density perturbations at $x>0$ are in counter phase with the variations at $x<0$. The basic feature of the density cross-correlations can be explained by taking the basic features to be a rotation of a compact density distribution in the parabolic potential well. When the center of mass of the plasma column is at some spatial position $\mathbf{r}^{\prime}$, there is a density enhancement there with respect to the time averaged density $\bar{n}(\mathbf{r})$, and a corresponding density depletion at the position $-\mathbf{r}^{\prime}$. With $\bar{n}(\mathbf{r})$ having an approximate Gaussian form, see Fig. A1, the fluctuating part $\widetilde{n}(\mathbf{r}, t)$ will at any time $t$ have an elongated form and its rotation in the parabolic potential well gives rise to time delays that increase with radial position as in Fig. 8 .

The basic differences between the space-time varying correlations of potential and density in Figs. 5 and 8 are explained by our simple model summarized in Section 4. By inspection of the correlations in Figs. 5 and 8 it seems evident that an assumption of the electrons being in local isothermal Boltzmann distribution, $\widetilde{n} / \bar{n} \approx e \phi / T_{e}$, is in error.

Figure 8. Cross-correlation between the floating potential from the fixed probe and the fluctuating plasma density detected by the moving probe (see Fig. 1).

The previous results for cross-correlations between the reference signal and density, potential and an electric field component should be considered with some caution: correlations between $\phi_{r}$ and $\widetilde{n}$ and between $\phi_{r}$ and $E_{y}$ do not contain information concerning correlations between $\widetilde{n}$ and $E_{y}$. This information, which is important for quantifying turbulent transport, has to be found by independent means.

Conspicuous features found by the correlation measurements can be summarized as: the auto-correlations (see Figs. 3, 4 and 7) demonstrate that the plasma fluctuations contain a significant harmonic component at a frequency corresponding the basic plasma rotation frequency. In addition there is a noise component with a short correlation time dominating the auto correlations at small time delays. Potential variations are nearly in phase for all $x$-positions at $y=0$. The variations in the potential signal are thus dominated by seemingly vertical motions. From the potential cross-correlations (see Fig. 5) we find that when the potential signal is maximum at the vicinity of the 


\section{Basic features of magnetized plasma torii}

reference probe it is near zero along the line $y=0$. Density fluctuations are in counterphase for $x>0$ and $x<0$. The temporal variations in density shows clear effects of the plasma rotation by an $x$-varying time delay, which is not found in the potential variations. We suggest a simple model that can account for most of these observations. When comparing analytical results with observations we bear in mind that the plasma center (i.e. the DC potential minimum) is slightly below $y=0$, see Fig. A1.

The product of the signals for density and $\mathbf{E} \times \mathbf{B} / B^{2}$-velocities gives the lowest order approximation to the plasma losses. Due to the different spatial phase relations of density and electric field the phase variation of their product, i.e. the plasma flux, will have a correspondingly complicated spatial variation.

\section{A simple analytical model}

The plasma in Blaamann and similar devices is complicated by being strongly fluctuating, inhomogeneous and anisotropic. Analytical models have been discussed $[1,6]$, giving insight into many of the basic plasma properties. Here we emphasize some of the features that we believe are important for the analysis to be discussed in the following. Two elements are given special attention, the average plasma rotation and the inhomogeneous magnetic field that gives rise to a polarization due to the $\nabla B \times \mathbf{B}$ and curvature drifts. The distinction between the electron and ion dynamics enters as a part of the analysis. Our model is thus based on two separate fluids, but rotation has interest also in single fluid, MHD, modeling of plasmas [12].

\subsection{Plasma polarization by $\nabla B \times \mathbf{B}$ and curvature drifts}

A parabolic DC potential variation in Blaamann was found for several parameter sets $[1,6,13,14]$ as also in other devices [15], except for cases with the discharge filaments placed near the wall of confining vessel. It may be worthwhile to estimate the relative importance of the rotation velocity and the bulk plasma drift induced by the plasma polarization due to the $\nabla B \times \mathbf{B}$ and curvature drifts.

To obtain a simple solvable model we assume that a parabolic approximation for the DC-potential with circular equi-potential lines is sufficiently accurate, giving

$$
\frac{E_{0}(\mathbf{r})}{B}=-\frac{E_{0}\left(r_{0}\right)}{B} \frac{\mathbf{r}}{r_{0}} .
$$

With little additional effort it is possible to generalize the model to elliptical equipotential lines, but this gives only limited additional physical information at the expense of lengthy mathematical expressions. By this parabolic potential approximation we have a nearly solid body $\mathbf{E}_{0} \times \mathbf{B} / B^{2}$-rotation of the plasma if we assume the magnetic field to be nearly constant in the plasma cross-section.

We also take the plasma column to have a circular cross-section with a uniform density $n_{0}$ inside the circular column and vanishing outside. A basic advantage of the suggested model is that the circular density cross-section retains its shape as it rotates 
with the angular velocity induced by (3). The radius of the circle turns out to be of minor importance for details in the analysis. We assume the space-time varying plasma density column to be strictly toroidally aligned at all times, an assumption supported by observations [13]. The $\nabla B \times \mathbf{B}$-velocity caused by the inhomogeneous magnetic field is assumed to be locally constant and in the $y$-direction. This will be appropriate for a long thin toroidal plasma column, as relevant also for the Blaamann plasma where the inner radius of the magnetic field coil is $18 \mathrm{~cm}$, see also an illustration presented elsewhere [1].

The basic equation of motion for the center-of-mass $\mathbf{R}_{i}(t)$ of the plasma ion component is then

$$
\begin{aligned}
\frac{d}{d t} \mathbf{R}_{i}(t)= & \frac{\mathbf{E}\left(\mathbf{R}_{i}(t), t\right) \times \mathbf{B}}{B^{2}} \\
& +\frac{1}{\Omega_{c i}} \frac{d}{d t} \frac{\mathbf{E}\left(\mathbf{R}_{i}(t), t\right)}{B}+U_{i} \widehat{\mathbf{y}}
\end{aligned}
$$

including $\mathbf{E} \times \mathbf{B}$ and polarization drifts together with the gxadient drift

$$
\begin{aligned}
\mathbf{U}_{i} & =\frac{W_{i}}{e B(x)} \frac{\mathbf{B} \times \nabla B}{B^{2}(x)} \\
& =\frac{W_{i}}{e B_{0} R_{0}} \widehat{\mathbf{y}}=\text { constant }
\end{aligned}
$$

with $W_{i} \equiv \frac{1}{2} M u_{t h i}^{2}$ being the average ion kinetic energy and $e>0$ is the ion charge. With $B_{0}$ being a reference magnetic field at the the major radius $R_{0}$, we have for the simple torus $\mathbf{B}=B_{0}\left(1+x / R_{0}\right)^{-1} \widehat{\mathbf{b}}$, with $B_{0}>0$. The unit vector $\widehat{\mathbf{b}}$ gives the direction of the magnetic field. The electron velocity $\mathbf{U}_{e}$ is found similarly by using the charge $-e$ and energy $W_{e}$. A curvature drift contributes to both $\mathbf{U}_{i}$ and $\mathbf{U}_{e}$ with a term like (5) apart from a numerical factor $[16,17]$. We take $U_{i}>0$ and $U_{e}>0$ in the following and give the direction of the bulk electron motion through the proper sign.

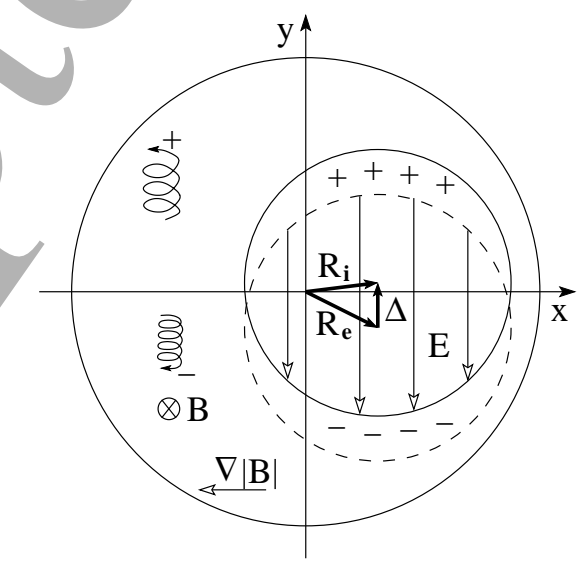

Figure 9. Schematic illustration of the polarization of a simple model for the Blaamann plasma, here with a circular cross-section and uniform density, ignoring the stationary parabolic potential well. The basic reference toroidal magnetic field points in the negative $\mathbf{z}$-direction in the figure, as indicated by $\otimes$, so that $\widehat{\mathbf{b}}=-\widehat{\mathbf{z}}$. 


\section{Basic features of magnetized plasma torii}

The electric field originates from two parts: one from $\mathbf{E}_{0}(r)$ given by (3), being imposed via the filament and therefore assumed constant in time, and another time varying part induced by polarization of the plasma. We find by an elementary calculation using a locally cylindrical approximation

$$
\mathbf{E}(\mathbf{r}, t)=-\frac{E_{0}\left(r_{0}\right)}{r_{0}} \mathbf{r}-\frac{1}{2} \frac{e n_{0}}{\varepsilon_{0}} \boldsymbol{\Delta}(t)
$$

where $\boldsymbol{\Delta}(t) \equiv \mathbf{R}_{i}(t)-\mathbf{R}_{e}(t)$ and $e>0$. Surface charges are created when the electrons are displaced slightly with respect to the ions. It is well known that these charges give rise to a constant electric field between the two lens-shaped parts of the cross-section, see Fig. 9, with the field direction being along $-\boldsymbol{\Delta}$. The factor $1 / 2$ originates from the local cylindrical geometry applicable when $r_{0} / R_{0} \ll 1$. The polarity of $\mathbf{E}_{0}(\mathbf{r})$ is given by the experimental conditions to point into the plasma. The electric fields and equi-potential lines are illustrated in Fig. 10. Since we have infinite density gradients on the edges of the electron and ion plasma columns, we can not impose any assumption of quasi neutrality. It is natural to assign a reference potential $\phi_{0}=0$ to the line $y=0$ in Fig. 10. This reference line will be moving as the plasma column moves.

The model implied by (4)-(5), with corresponding equations for the electrons and (6) can be analyzed as an initial value problem. It will remain valid until $\Delta$ becomes noticeably larger than $\lambda_{D e}$. We find that for relevant plasma parameters this will take several rotation periods $2 \pi / \Omega_{0}$. At larger times the two lens shaped regions in Fig. 9 become distorted, and this limit is not included in the analysis.

The results of the present section with a "top hat" plasma density variation can be generalized, as illustrated in the following, to include a small vertical magnetic field component as well as ion-neutral collisions. As long as we retain the strictly toroidal magnetic field it is, however, possible to give a numerical solution for an arbitrary density profile, and also to take into account the magnetic field more accurately in the analysis. When this feature is retained, the plasma flow becomes compressible with $\nabla \cdot\left[\mathbf{E}(\mathbf{r}, t) \times \mathbf{B}(\mathbf{r}) / B^{2}(\mathbf{r})\right] \neq 0$. This analysis is presented in Appendix B.

\subsection{Consequences of a vertical magnetic field and electron collisions}

When operating the Blaamann device it was often found to be an advantage to impose a small vertical magnetic field component $B_{y}$. This gave a stable and less noisy discharge. Following [18] we can suggest a phenomenological model accounting for some basic features of the vertical B-component. When $B_{y}$ is small compared to the axial or toroidal magnetic field component, we can retain the two-dimensional model for the ion dynamics used before, using the argument that $u_{i \|} \ll u_{i \perp}$ in terms of the B-parallel and perpendicular ion velocities. For the electron motion, it is often found that the electron mobility along magnetic field lines is so large that $U_{e \|} \gg U_{e \perp}$ in terms of the B-parallel and perpendicular electron velocities. In this limit the electron motion will be controlled by collisions between electrons and ions or neutrals. To describe the effect 
Figure 10. Illustration of electric fields and equi-potential lines for a polarized model "top-hat" density distribution. Analytical expressions for the electric fields and potential are given elsewhere [18].

of collisions on the electron motion we use

$$
-T_{e} \frac{\partial n}{\partial s}-n e E_{s}-n m \nu u_{e s} \approx 0 \text {, }
$$

where we introduced $s$ as the coordinate along the tilted magnetic field lines. The subscript $s$ specifies electric field and electron fluid velocity components along the tilted magnetic field lines. We introduced $\nu$ as an electron collision frequency and $T_{e}$ is a constant electron temperature. Electron inertia has been ignored due to the smallness of the electron mass $m$. This limit explicitly assumes a constant electron mobility, and therefore $\nu \neq 0$.

The case where the electron mobility is very large, it is often assumed the electron component can be taken to be Boltzmann distributed at all times. We find this limit to be marginally relevant and take instead a constant mobility to give $u_{e s} \approx-e E_{s} /(\nu m)$ since $\partial n / \partial s=0$ for the present top-hat plasma density distribution (except at the edges). The vertical component of the electron velocity is found to be $u_{e y} \approx u_{e s} \sin \theta \approx$ $-(e / \nu m) E_{s} \sin \theta \approx-(e / \nu m) \mathbf{E} \cdot \widehat{\mathbf{y}} \theta^{2}$. One $\theta$-contribution arises from the field aligned electric field that also has a vertical component $E_{y}$. The electron drift in the $\widehat{\mathbf{y}}$-direction is a combination of the $\nabla B \times \mathbf{B}$-velocity and $u_{e y}$. Although the present "top-hat" model ignores the contribution from $\partial n / \partial s$, we note that by relaxing the condition of a constant plasma density and for a given $\mathbf{E}$ (i.e. a given $\boldsymbol{\Delta}$ ), the short-circuiting electron velocity $u_{e y}$ will be enhanced when $\partial n / \partial y$ has the same sign as $E_{y}$ and reduced for the opposite sign. The consequences of this effect will vary with position in the $x-y$ plane. As a result, the plasma density column will in general lose the local cylindrical symmetry in actual experiments.

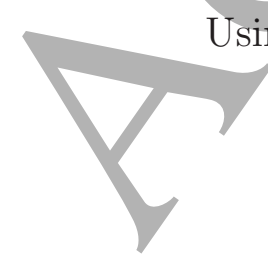

Using the expression for $\mathbf{E}(\mathbf{r}, t)$ given by (6) we find

$$
\begin{aligned}
\frac{d \mathbf{R}_{e}}{d t}= & -\mathbf{R}_{e}(t) \times \mathbf{B} \frac{E_{0}}{B^{2} r_{0}}-\frac{1}{2} \frac{e n_{0}}{\varepsilon_{0} B^{2}} \boldsymbol{\Delta}(t) \times \mathbf{B} \\
& +\left(\frac{1}{2} \frac{e^{2} n_{0}}{\varepsilon_{0} m \nu} \theta^{2} \boldsymbol{\Delta}(t) \cdot \widehat{\mathbf{y}}-U_{e}\right) \widehat{\mathbf{y}} .
\end{aligned}
$$




\section{Basic features of magnetized plasma torii}

The limit $\nu \rightarrow 0$ is not applicable here. We take $U_{e}>0$ and give the direction of the bulk electron motion through the - sign.

In general we have $U_{i} \neq U_{e}$ because of different ion and electrontemperatures. Note that the electric fields determining the motion of the electron and ion components, respectively, are to be obtained at $\mathbf{R}_{i}(t)$ and $\mathbf{R}_{e}(t)$, respectively, so the two contributions need not cancel by subtraction of the two expressions for $\mathbf{R}_{i}(t)$ and $\mathbf{R}_{e}(t)$. The spatial variation of the magnetic field is included via the last terms in (4) and (8) so $B$ is taken constant in the remaining terms for simplicity. The vertical magnetic field is approximately constant, while the toroidal magnetic field varies with $x$ in a more detailed representation. A complete model that takes into account the entire spatial variation of the magnetic field will have $\theta=\theta(x)$.

The parabolic potential well gives rise a rotation with frequency $\Omega_{0} \equiv\left|E_{0} /\left(B r_{0}\right)\right|$, introducing a natural reference time for the variations. In terms of the normalized time $\tau \equiv t \Omega_{0}$, the resulting expression for the ions becomes

$$
\begin{aligned}
\left(1+\frac{\Omega_{0}}{\Omega_{c i}}\right) \frac{d \mathbf{R}_{i}(\tau)}{d \tau}=-\mathbf{R}_{i}(\tau) \times \widehat{\mathbf{b}} \\
-\frac{1}{2}\left(\varepsilon_{r}-1\right) \frac{\Omega_{c i}}{\Omega_{0}} \Delta(\tau) \times \widehat{\mathbf{b}} \\
\quad-\frac{1}{2}\left(\varepsilon_{r}-1\right) \frac{d \boldsymbol{\Delta}(\tau)}{d \tau}+\frac{U_{i}}{\Omega_{0}} \widehat{\mathbf{y}}
\end{aligned}
$$

and for the electrons

$$
\begin{aligned}
& \frac{d \mathbf{R}_{e}(\tau)}{d \tau}=-\mathbf{R}_{e}(\tau) \times \widehat{\mathbf{b}} \\
&- \frac{1}{2}\left(\varepsilon_{r}-1\right) \frac{\Omega_{c i}}{\Omega_{0}} \Delta(\tau) \times \widehat{\mathbf{b}} \\
& \quad+\left(\frac{1}{2} \frac{\omega_{p e}^{2}}{\nu} \theta^{2} \Delta(\tau) \cdot \widehat{\mathbf{y}}-U_{e}\right) \frac{1}{\Omega_{0}} \widehat{\mathbf{y}} .
\end{aligned}
$$

The vectors $\mathbf{R}_{i}, \mathbf{R}_{e}$ and $\Delta$ are explained in Fig. 9. We introduced [19] the relative dielectric constant $\varepsilon_{r} \equiv 1+n_{0} M / \varepsilon_{0} B^{2}=1+\left(\Omega_{p i} / \Omega_{c i}\right)^{2}$. The ion mass appears explicitly through the ion cyclotron frequency due to the inclusion of the ion polarization drift. We have $U_{e} \approx\left|W /\left(e B_{0} R_{0}\right)\right| \approx 55 \mathrm{~m} \mathrm{~s}^{-1}$ for $5 \mathrm{eV}$ electrons, while for the colder ions we estimate $U_{i} \approx 0.6 \mathrm{~m} \mathrm{~s}^{-1}$, i.e. $U_{e} \gg U_{i}$. For typical plasma densities near the center in Blaamann we find $\epsilon_{r} \approx 3 \times 10^{3}$. The coefficient $\left(1+\Omega_{0} / \Omega_{c i}\right)$ on the left side of (9) accounts for a polarization due to a difference in rotation frequency for the ion and electron components found when the finite ion inertia is included [9, 15, 20]. The first terms on the right hand sides of (9) and (10) originate from the externally imposed potential well, so these terms are ignored for the case with $E_{0}=0$.

The analysis summarized here does not specify the nature of the collisions (electrons-ions or electrons-neutrals). As far as the electron momentum relaxation is concerned, the difference enters through the cross-sections which are needed for calculating $\nu$. The distinction between the two collision processes mentioned is found in 1) momentum exchange with the ions, and 2) build-up of an electric field. By 


\section{Basic features of magnetized plasma torii}

assuming that the dominant ion velocity is in the direction perpendicular to $\mathrm{B}$ with a magnitude $E / B$ we ignore effect 1 ). A similar assumption is found in the so-called Hasegawa-Wakatani model for weakly collisional electrostatic drift waves [21], where only viscosity due to ion-ion collisions is retained. The effect 2) originates from the distinction between collisional diffusion caused by electron-ion collisions as compared to the case with collisions between charged particles and neutrals. In the former case the plasma remains neutral since electron-ion collisions will not cause charge separation. In the latter case a steady state electric field will build up [22], with a magnitude of approximately $T_{e}+T_{i}$ measured in $\mathrm{eV}$ divided by the scale length of the density gradient in the direction perpendicular to $\mathbf{B}$. Since the magnitude of this electric field will be much smaller than $E_{0}$, its inclusion will be of little consequence. We therefore argue that $\nu$ can denote the collision frequency between electrons-ions or electrons-neutrals with no need for distinction.

\section{Solutions of the basic equations}

In the present section we present results derived from the expressions summarized in Section 4. For illustration we include some limiting cases, i.e. also one without a stationary potential well.

\subsection{Simple case with $E_{0}=0$ and $\theta=0$}

First we can make a simple reference analysis, ignoring the parabolic potential by setting $E_{0}=0$ and assuming a simple toroidal magnetic field without vertical magnetic field component. By subtracting (4)/and (8) with the terms originating from $E_{0}$ on the right hand sides discarded, an ordinary differential equation is obtained for $\Delta(t)=\mathbf{R}_{i}(t)-\mathbf{R}_{e}(t)$

$$
\frac{d}{d t} \boldsymbol{\Delta}(t)=2 \frac{U_{i}+U_{e}}{1+\varepsilon_{r}} \widehat{\mathbf{y}} .
$$

Since the present problem has no bulk rotation, and thus no normalizing $\Omega_{0}$, we use physical time here.

The vector $d \boldsymbol{\Delta} / d t$ in (11) is constant. With the present simplified assumptions, the relative displacement of electrons and ions therefore increases monotonically. The electric field produced by the separation accelerates the plasma in the radial direction, until the increase in $\Delta$ is arrested when the plasma reaches the walls of the confining toroidal vessel.

To find the acceleration of the bulk plasma we use the average position $\mathbf{R}_{p}(t) \equiv$ $\left(\mathbf{R}_{i}(t)+\mathbf{R}_{e}(t)\right) / 2$. By adding and then differentiating (4) and (8) we have by the given assumptions [18]

$$
\begin{aligned}
\frac{d^{2} \mathbf{R}_{p}}{d t^{2}} & =\frac{e n_{0}}{\varepsilon_{0} B} \frac{U_{i}+U_{e}}{1+\varepsilon_{r}} \widehat{\mathbf{x}} \\
& =\Omega_{c i} \frac{\varepsilon_{r}-1}{\varepsilon_{r}+1}\left(U_{i}+U_{e}\right) \widehat{\mathbf{x}}
\end{aligned}
$$


Basic features of magnetized plasma torii

$$
\approx \Omega_{c i}\left(U_{i}+U_{e}\right) \widehat{\mathbf{x}}=\text { const }
$$

since $d^{2} \boldsymbol{\Delta} / d t^{2}=0$. In the limiting case for large $n_{0}$ as given in (12), appropriate for the central parts of the Blaamann plasma [9], we find $d^{2} \mathbf{R}_{p} / d t^{2}$ to be independent of plasma density. The induced polarization electric field is $E \approx e B^{2}\left(U_{i}+U_{e}\right) t / M$ in the limit of large $\varepsilon_{r}$. Without any potential well, i.e., without plasma rotation, the plasma is lost through a constant acceleration in the direction of the major radius. This result accounts for the well known lack of equilibrium for a simple magnetized toroidal plasma $[1,6]$. For low density plasmas we find $d^{2} \mathbf{R}_{p} / d t^{2} \approx \Omega_{p i}^{2}\left(U_{i}+U_{e}\right) / \Omega_{c i} \widehat{\mathbf{x}}$, indicating that a low density plasma is better confined than one with high density. The difference can be explained by noting that $\varepsilon_{r}$ increases monotonically with plasma density from the vacuum value $\varepsilon_{r}=1$, while the surface charge density at the boundary of the "top-hat" profile is directly proportional to the plasma density. As the plasma density vanishes we have $\varepsilon_{r} \rightarrow 1$ and $d^{2} \mathbf{R}_{p} / d t^{2} \rightarrow 0$.

A qualitative argument gives that the cross-section of a plasma with inhomogeneous density, with density large in the center and decreasing outwards, will be deformed to a cross-section with a horse-shoe shape [19] as it expands by being accelerated in the direction of the major radius of the torus. For the present helium discharge in the given magnetic field we find $\varepsilon_{r} \gg 1$ in most of the plasma column, except for the edges close to the walls. The density dependence of $\varepsilon_{r}$ is important for applications of the model $[18]$.

\subsection{General case with $E_{0} \neq 0$ but $\theta=0$}

To account for the basic plasma rotation induced by the externally imposed parabolic well we now take $E_{0} \neq 0$. The basic equation in its general form has surprisingly complicated analytical solutions. We therefore consider the simple limit where the rotation frequency of the plasma is much smaller than $\Omega_{c i}$. Introducing the normalized time $\tau \equiv t \Omega_{0}$ we have

$$
\frac{1}{2}\left(1+\varepsilon_{r}\right) \frac{d}{d \tau} \Delta(\tau)=-\Delta(\tau) \times \widehat{\mathbf{b}}+\frac{U_{i}+U_{e}}{\Omega_{0}} \widehat{\mathbf{y}}
$$

with solution $\left.\Delta_{x}(\tau)=2 \sin ^{2}\left(\tau /\left(1+\varepsilon_{r}\right)\right)\right)\left(U_{i}+U_{e}\right) / \Omega_{0}$ and $\Delta_{y}(\tau)=\sin \left(2 \tau /\left(1+\varepsilon_{r}\right)\right)\left(U_{i}+\right.$ $\left.U_{e}\right) / \Omega_{0}$ fulfilling $\left(\Delta_{x}(0), \Delta_{y}(0)\right)=(0,0)$. The problem now has two time scales: a fast scale $\Omega_{0}^{-1}$ and a slow scale $\left(1+\varepsilon_{r}\right) \Omega_{0}^{-1}$.

With $E_{0} \neq 0$ it is seen that now $|\Delta(t \tau)|$ is finite for all times, implying that a uniform plasma rotation has a stabilizing effect on the plasma polarization. For plasma conditions relevant in the Blaamann experiment we find a length scale $\left(U_{i}+U_{e}\right) / \Omega_{0}<$ $10^{-3} \mathrm{~m}$, which is small, albeit larger than the Debye length.

Inserting $\Delta_{x}(\tau)$ and $\Delta_{y}(\tau)$ in the expression for $\mathbf{R}_{p}(\tau)$ found by adding (9) and (10), the result is

$$
\begin{aligned}
& \frac{d}{d \tau} \mathbf{R}_{p}(\tau)=-\mathbf{R}_{p}(\tau) \times \widehat{\mathbf{b}} \\
& \quad-\frac{1}{2}\left(\varepsilon_{r}-1\right) \frac{\Omega_{c i}}{\Omega_{0}} \Delta(\tau) \times \widehat{\mathbf{b}}
\end{aligned}
$$


Basic features of magnetized plasma torii

$$
-\frac{1}{4}\left(\varepsilon_{r}-1\right) \frac{d \boldsymbol{\Delta}(\tau)}{d \tau}+\frac{U_{i}-U_{e}}{\Omega_{0}} \widehat{\mathbf{y}} .
$$

For short times where the plasma polarization is small, the two terms containing $\Delta(\tau)$ on the right hand side can be ignored. With $\left(R_{p x}(0), R_{p y}(0)\right)=(0, A)$ the solution is $R_{p x}(\tau) \approx 2 \sin ^{2}(\tau / 2)\left(U_{i}-U_{e}\right) / \Omega_{0}+A \sin (\tau)$ and $R_{p y}(\tau) \approx \sin (\tau)\left(U_{i}-U_{e}\right) / \Omega_{0}+A \cos (\tau)$, corresponding to a rotation and a displacement of the plasma column in the parabolic well. For large times where $\Delta(\tau)$ contributes substantially we also have analytical results for $\mathbf{R}_{p}(\tau)$, but they are very lengthy and will not be reproduced here.

To the present approximation both $|\boldsymbol{\Delta}(\tau)|$ and $\left|\mathbf{R}_{p}(\tau)\right|$ will be bounded. In order for the result to have practical applicability we need, however, more restrictive conditions satisfied, namely that the plasma is positioned inside the confining vessel with radius $\sim r_{0}$, giving $|\boldsymbol{\Delta}(\tau)| \ll r_{0}$ and $\left|\mathbf{R}_{p}(\tau)\right| \ll r_{0}$ for all times. The first condition is trivially satisfied. We find that for large plasma densities, i.e., $\varepsilon_{r} \gg 1$ the maximum displacement of the plasma column as given by $\left|\mathbf{R}_{p}(\tau)\right|$ scales approximately as $\sim\left(U_{i}+U_{e}\right) \varepsilon_{r} / \Omega_{0}$ which can be $1-10 \mathrm{~m}$ for Blaamann conditions, i.e. much larger than the minor radius of the confining vessel. Although the solution is mathematically bounded, the plasma will be lost to the wall, but it takes a few rotations in the parabolic plasma potential well before this happens. Numerical solutions for realistic Blaamann parameters are shown in Fig. 11. The model has no a priori assumption of quasi-neutrality, but the separation between the electron and the ion component remains within the electron Debye length scale nonetheless. Figure 11 indicates a vertical motion of approximately $35 \mathrm{~cm}$ within a time $2 \pi / \Omega_{0} \approx 0.11 \times 10^{-3} \mathrm{~s}$. This corresponds to a vertical velocity component of $3.1 \times 10^{3} \mathrm{~m} \mathrm{~s}^{-1}$. This is within a factor 2 with the results for a downward vertical velocity of $1.5 \times 10^{3} \mathrm{~m} \mathrm{~s}^{-1}$ found in Section 3.1. Given the approximations made in the analysis, we consider this agreement to be satisfactory.

Information supporting the results in Fig. 11 is presented in Appendix B for the limit of $\varepsilon_{r} \gg 1$. These results have no constraints on the spatial variation of the instantaneous plasma density. We find good qualitative agreement between the results concerning the rotation and slow downward drift of the plasma column. Although the simple top-hat model is restrictive, we find that this agreement with a more general model gives confidence for the use of the simplified model for other problems.

The present conditions ean be stabilized within a realistic plasma cross-section when $\Omega_{0} \geq \Omega_{c i}$ and thus the coefficient for $d \mathbf{R}_{i} / d \tau$ in (9) becomes significantly enhanced. This can be achieved by heavy ions and large $E_{0}$, but this limit has not been studied in any detail in Blaamann. Such conditions have been realized in linear devices [23].

We observe a net downward drift of the plasma due to the combined effects of rotation and polarization electric field. Due to this break in up-down symmetry, probe $\mathrm{CH} 2$ is partially shadowed by probe $\mathrm{CH} 1$, explaining the observed difference in fluctuation level described in Section 2. 
Basic features of magnetized plasma torii
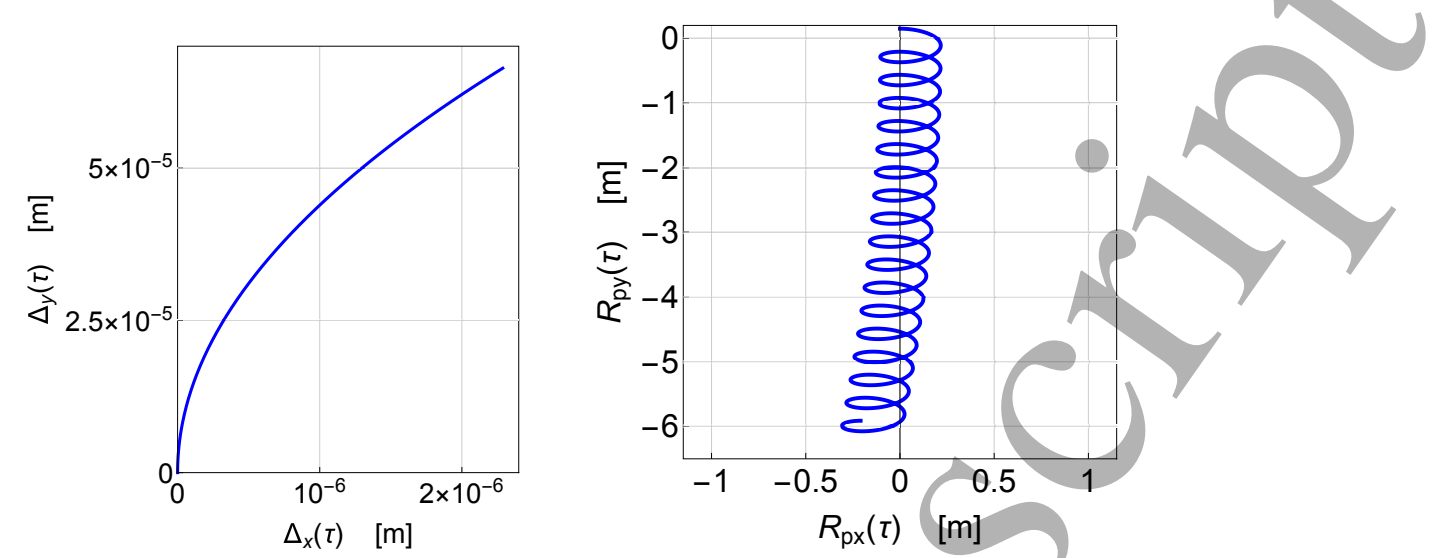

Figure 11. Illustration of the time evolutions of $\boldsymbol{\Delta}(\tau)$ and $\mathbf{R}_{p}(\tau)$ in a plane perpendicular to $\mathbf{B}$ for the case where $\theta=0$. The time interval shown is $\tau \in$ $\{0,17\} 2 \pi / \Omega_{0}$. The plasma parameters are taken from Table A1. Due to the short time interval shown it appears as if the average trajectory is nearly vertical, where in reality it is a circle with large radius as seen/when extending the time duration of the calculation.

\subsection{Consequences of a small vertical magnetic field component, $\theta \neq 0$}

A small vertical magnetic field component is now included. This will only affect the electron motion: the ion dynamics can still be considered in a plane perpendicular to the torus axis. We again ignore the small $\Omega_{0} / \Omega_{c i}$ correction on the left side of (9). By (9) and (10) the separation $\boldsymbol{\Delta}$ we find

$$
\begin{aligned}
& \frac{1}{2}\left(\varepsilon_{r}+1\right) \frac{d \boldsymbol{\Delta}(\tau)}{d \tau}=-\boldsymbol{\Delta}(\tau) \times \widehat{\mathbf{b}} \\
& \quad-\frac{1}{2} \frac{\omega_{p e}^{2}}{\Omega_{0} \nu} \theta^{2} \boldsymbol{\Delta}(\tau) \cdot \widehat{\mathbf{y}} \widehat{\mathbf{y}}+\frac{U_{i}+U_{e}}{\Omega_{0}} \widehat{\mathbf{y}},
\end{aligned}
$$

and the average position $\mathbf{R}_{p}$

$$
\begin{aligned}
\frac{d \mathbf{R}_{p}}{d \tau}=-\mathbf{R}_{p}(\tau) \times \widehat{\mathbf{b}} \\
\quad-\frac{1}{2}\left(\varepsilon_{r}-1\right) \frac{\Omega_{c i}}{\Omega_{0}} \Delta(\tau) \times \widehat{\mathbf{b}} \\
+\frac{1}{4} \frac{\omega_{p e}^{2}}{\Omega_{0} \nu} \theta^{2} \boldsymbol{\Delta}(\tau) \cdot \widehat{\mathbf{y}} \widehat{\mathbf{y}} \\
-\frac{1}{4}\left(\varepsilon_{r}-1\right) \frac{d \boldsymbol{\Delta}(\tau)}{d \tau}+\frac{U_{i}-U_{e}}{\Omega_{0}} \widehat{\mathbf{y}} .
\end{aligned}
$$

The last term accounts for the $\nabla B \times \mathbf{B}$-drifts in the $y$-direction. For increasing $\boldsymbol{\Delta}$ these terms are counteracted by the terms containing $\theta^{2}$. This reduction is due to the short-cireuiting effect of the electrons as they move along the slightly tilted magnetic field linés. Numerical solutions for realistic Blaamann parameters are shown in Fig. 12. By comparing with Fig. 11 we find it interesting to see how strong an effect even a small vertical magnetic field component can have. The explanation is found in the high 


\section{Basic features of magnetized plasma torii}

electron mobility along magnetic field lines, which gives a significant vertical electron velocity component even for small $B_{y}$.
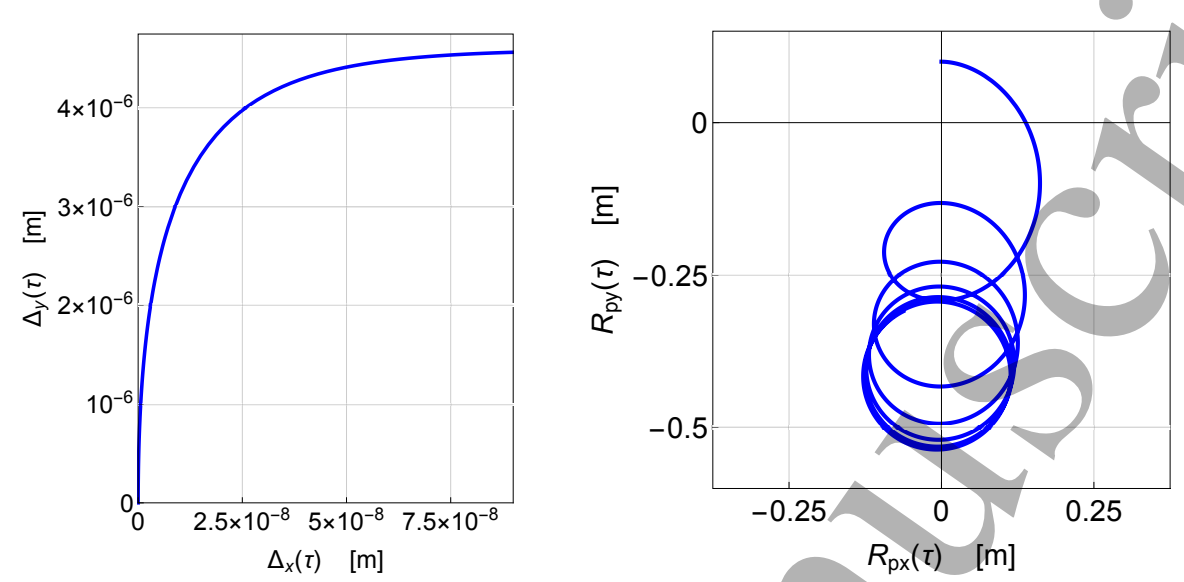

Figure 12. Illustration of the time evolutions of $\boldsymbol{\Delta}(\tau)$ and $\mathbf{R}_{p}(\tau)$ in a plane perpendicular to $\mathbf{B}$, here for the case where $\theta \neq 0$. The time interval shown is $\tau \in\{0,6\} 2 \pi / \Omega_{0}$. The parameters are taken from Table A1. Compare with Fig. 11 .

Equations for $\Delta_{x}(\tau)$ and $\Delta_{y}(\tau)$ are readily found from (15). We find a limiting value for $\tau \rightarrow \infty$ as $\Delta_{y}=2\left(U_{i}+U_{e}\right) \nu /\left(\theta \omega_{p e}\right)^{2}$, while $\Delta_{x}$ increases slowly with $\tau$.

By Fig. 12 we note that a quasi-stationary condition is reached within 5 - 6 rotation periods $2 \pi / \Omega_{0}$. Within this time limit we have $\Delta_{x} \ll \Delta_{y}$, implying that $\Delta$ is nearly vertical, so that all equi-potential lines are nearly horizontal and $\| \widehat{\mathbf{x}}$. This result is in qualitative agreement with the observations summarized in Fig. 5.

\subsection{Effects of ion-neutral collisions}

To account for ion collisions we modify the ion dynamics by including a collisional friction with frequency $\hat{\nu}_{i}$ entering in the analytical form

$$
\begin{aligned}
& \left(1+\frac{1}{\Omega_{c i}} \frac{E_{0}\left(r_{0}\right)}{B r_{0}}\right) \frac{d^{2} \mathbf{R}_{i}}{d t^{2}}= \\
& -\frac{d \mathbf{R}_{i}}{d t} \times \mathbf{B} \frac{E_{0}\left(r_{0}\right)}{B^{2} r_{0}}-\frac{1}{2} \frac{e n_{0}}{\varepsilon_{0} B^{2}} \frac{d \boldsymbol{\Delta}}{d t} \times \mathbf{B} \\
& -\frac{1}{2} \frac{e n_{0}}{\varepsilon_{0} B \Omega_{c i}} \frac{d^{2} \boldsymbol{\Delta}}{d t^{2}}-\nu_{i} \frac{d \mathbf{R}_{i}}{d t} .
\end{aligned}
$$

At first sight it seems that $U_{i}=$ constant has vanished from the expressions, but it contributes through the initial conditions. The last term in (17) enters as a standard friction term. The equation can be integrated once with respect to $t$. The electron equation (10) is unchanged.

In terms of normalized time the ion equation takes the form

$$
\begin{gathered}
\frac{d \mathbf{R}_{i}}{d \tau}=-\mathbf{R}_{i} \times \widehat{\mathbf{b}}-\frac{1}{2}\left(\varepsilon_{r}-1\right) \frac{\Omega_{c i}}{\Omega_{0}} \boldsymbol{\Delta} \times \widehat{\mathbf{b}} \\
-\frac{1}{2}\left(\varepsilon_{r}-1\right) \frac{d \boldsymbol{\Delta}}{d \tau}-\frac{\nu_{i}}{\Omega_{0}} \mathbf{R}_{i}+\frac{U_{i}}{\Omega_{0}} \widehat{\mathbf{y}},
\end{gathered}
$$




\section{Basic features of magnetized plasma torii}

where a small correction $\Omega_{0} / \Omega_{c i}$ is ignored. The polarization equation, accounting for the time evolution of $\boldsymbol{\Delta}$, becomes

$$
\begin{aligned}
& \frac{1}{2}\left(\varepsilon_{r}+1\right) \frac{d \boldsymbol{\Delta}(\tau)}{d \tau}=-\boldsymbol{\Delta}(\tau) \times \widehat{\mathbf{b}} \\
& \quad-\frac{1}{2} \frac{\omega_{p e}^{2}}{\Omega_{0} \nu} \theta^{2} \Delta_{y}(\tau) \widehat{\mathbf{y}}+\frac{U_{i}+U_{e}}{\Omega_{0}} \widehat{\mathbf{y}}-\frac{\nu_{i}}{\Omega_{0}} \mathbf{R}_{i} .
\end{aligned}
$$

Illustrative short time numerical solutions for the coupled equations (18) and (19) are shown in Fig. 13. The model summarized here assumes that the electron collisions are only important by controlling the electron flow along magnetic field lines. We note that for increasing times, the $\boldsymbol{\Delta}$ vector is "tilting" and $\Delta_{y}$ is reduced. At these times, collisions will be important also for the electron dynamics in the $x-y$ plane, and the electron collision model has to be generalized. This can not be done properly within the given top-hat model.

The ion collision model assumes that all ions are affected exactly the same way by the collisions. The statistical or random nature of the collisions will give rise to a slow diffusion of the plasma column. At late times the original top-hat model will be eroded by collisions to take a near Gaussian density distribution. This asymptotic limit is not covered by the analysis as our results are only valid for short times.

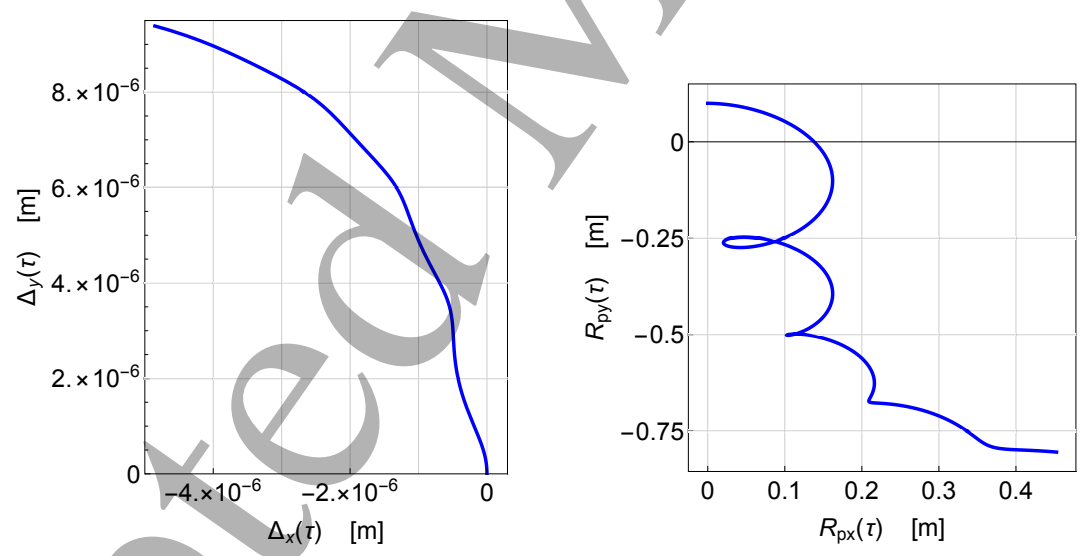

Figure 13. Illustration of the short time evolutions of $\boldsymbol{\Delta}(\tau)$ and $\mathbf{R}_{p}(\tau)=\mathbf{R}_{i}(\tau)-\boldsymbol{\Delta} / 2$ in a plane $\perp \mathbf{B}$, here for the case where $\theta \neq 0$ and including ion-neutral collisions. For illustration we use here a small collision frequency of $\nu_{i}=100 \mathrm{~s}^{-1}$. The other data are taken from Table A1. The time interval shown is $\tau \in\{0,4\} 2 \pi / \Omega_{0}$.

Going into more detail we note that the collision frequencies listed in Table A1 refer to momentum losses. Taking an electron starting with $U_{\perp} \gg U_{\|}$we will find the $\nabla B \times \mathbf{B}$ drift to be dominating. After a collision it may be so that now $U_{\|} \gg U_{\perp}$ and in this case it will be the curvature drift dominating. The average drift velocity remains, however, to be in the same direction and within a factor 2 the same magnitude as before the collision [16]. It will take approximately $M / m$ collisions to change the electron energy significantly.

Some robust results emerge from the analysis summarized in the present section: 
1) The plasma column rotates in the DC-potential well with a frequency close to $\Omega_{0}$.

2) The polarization vector of the plasma is nearly vertical at all times, implying that to lowest approximation the electrostatic equi-potential curves are parallel to the $x$-axis at all times. These contours will move in the $y$-direction. This observation refers to the top-hat model, but will remain a good approximation also for more realistic cases. A fixed probe will thus detect a dominant frequency $\Omega_{0}$ for fluctuations in both potential and density. For the idealized top-hat model the polarization electric field will be the same at any position inside the plasma. Ion collisions will tend to tilt the direction of the polarization vector $\boldsymbol{\Delta}$ so that $\Delta_{x}$ becomes slightly negative, while it is positive when ion collisions are ignored. The experimental results summarized in Fig. 5 indicate that the potential contours are tilted slightly in the predicted direction, at least for $x>0$.

3 ) During its motion, the plasma column will spend more time for $x>0$ than for $x<0$, i.e. the plasma column will on average be displapced towards the low magnetic field side of the cross-section.

\section{Discussions and Conclusion}

We presented results related to the performance of a toroidally magnetized plasma. In its basic configuration, the plasma has no equilibrium and will be lost to the chamber wall by the $\mathbf{E} \times \mathbf{B} / B^{2}$-drift due to the polarization electric field induced by the $\nabla B \times \mathbf{B}$ particle motion. The basic configuration can be modified by externally imposed steady state electric and magnetic fields. We considered the effects of a parabolic potential well and the resulting electric field $\mathbf{E}=\mathbf{r} E_{0} / r_{0}$ where $r_{0}$ is the minor radius of the torus. Also a small vertical magnetic field component are investigated in a plasma with neutral collisions.

Analytical results based on a simple top-hat model for the plasma density demonstrates that for the simple toroidal magnetic field, the bulk plasma rotation induced by the steady state radial electric field has a stabilizing effect by confining the plasma within a finite radius that scales approximately as $\left(U_{i}+U_{e}\right) \varepsilon_{r} / \Omega_{0}$. In terms of basic plasma and device parameters the scaling of the confining radius is proportional to $\left(r_{0} / R_{0}\right) \operatorname{Tn} M /\left(B_{0}^{2} E_{0}\right)$, indicating that cold plasmas with light ions in devices with large aspect ratios, $R_{0} \gg r_{0}$, are most easily confined by rotation. The basic features of the top-hat model were confirmed by a more general model presented in Appendix B for the limit of $\varepsilon_{r} \gg 1$. Although the simple top-hat model is restrictive, we find that this agreement with a more general model gives confidence for the use of the model for other and more general problems.

For realistic plasma parameters it turns out that the plasma rotation is not sufficient for a complete stabilization. The conditions are improved by a weak vertical magnetic field that gives a partial short circuiting of the polarization electric field in a plasma with neutral collisions. Also these effects were discussed and illustrated by solutions of the analytical expressions. 


\section{Basic features of magnetized plasma torii}

While an improved confinement (or rather reduction of losses) by the plasma rotation can be difficult to quantify, there are other aspects of the model that can be analysed. The plasma rotation and polarization by the $\nabla B \times \mathbf{B}$-particle drifts have different consequences for the perturbations in plasma density and potential. For reference, we obtained data for the time variations of the fluctuations of the plasma density and the potential as detected by a movable multi-probe, see Fig. 1. The fluctuations represent the difference between the instantaneous variations on the plasma and the time averaged values shown in Fig. A1. The results are presented in terms of auto-correlations for the signal, as well as cross-correlations with respect to a fixed reference probe, see Fig. 1. The analysis demonstrates that the fluctuating equi-potential lines are nearly horizontal, i.e., nearly parallel to the $x$-axis in Figs. 1 and 9 . This is in good agreement with observations summarized in Fig. 5. The perturbation of the plasma density with respect to the time average shown in Fig. A1 has an elongated "banana" shaped spatial variation. The rotation of this form gives a pronounced increase in time delays in the density variation for increasing radial positions, see Fig. 8, to be compared with the potential signal in Fig. 5. The spatial variations of the potential and density perturbations are significantly different at any given time. The analysis was extended to cover qualitatively also the corresponding variations of the fluctuating vertical electric field component and the resulting time varying low frequency plasma flux in the $x$ direction. This plasma transport has also a high frequency component originating from small-scale fluctuations induced by instabilities on the edge of the plasma. Many studies emphasize the plasma losses due to these effects.

When modeling the combined $\nabla B \times \mathbf{B}$ and curvature drifts we used expressions where the particle energies were associated with their thermal energies. An accurate model will retain the explicit particle energy dependence of for instance the $\nabla B \times \mathbf{B}$ drifts as e.g. $U_{e} \approx \frac{1}{2} m U_{\perp}^{2} /\left(e R_{0} B\right)$. This means that there will be a tendency for the most energetic particles, to drift faster than the slower ones for the present moderately collisional plasma, see Table A1. As a consequence, we will be likely to find an uneven particle energy distribution across the plasma column in the vertical direction, the most energetic ions at the top and for electrons near the bottom, with the spatial energy distribution following at least approximately the potential variation, see Fig. 9 and also Fig. 12. Since theion drift velocities are moderate compared to the electron velocities due to the small average ion energy, see Table A1, the effect will in our case be pronounced for the electron energy distribution only. The spatial distribution of the electron energy density will be discussed in more detail in an accompanying paper.

As well known, the plasma boundary of the simple model used here is not stable $[2,24]$. If the plasma boundary is perturbed by a "bulge" on the low magnetic field side, this perturbation will increase in magnitude for both convex and concave perturbations. The high magnetic field side is stable in this respect. Consequently we expect that the simple plasma density models discussed in Section 4 will be disturbed by randomly varying density and potential perturbations. The foregoing analytical discussions, supported by experimentally obtained correlations, demonstrated that the 


\section{Basic features of magnetized plasma torii}

space-time variation of density, temperature and electric fields in Blaamann and similar discharge plasmas develop differently with different relative spatial phase relations. As a consequence we expect fluxes of plasma density and energy density losses to be significantly different.

One important "bi-product" of the analytical model presented here is the illustration of problems arising by use of an AC coupling of the detecting systems as done in many experiments. Concerning the potential we can prescribe any reference level in space, $\phi \rightarrow 0$ at $|\mathbf{r}| \rightarrow \infty$ for instance. The AC coupling will, however, be equivalent to prescribing $\langle\phi\rangle=0$ for all positions. Consequently, we will find also $\langle\mathbf{E}\rangle=0$ at any position, since this electric field is found by a potential difference divided by a probe separation. As our model (illustrated in e.g. Fig. 10) demonstrates, this constraint on the electric field can be overly restrictive, and the results should be interpreted with care. One basic feature of the model is its prediction of a generally positive plasma potential for $y>0$ and generally negative potential for $y<0$, resulting in a vertical polarization electric field component with a non vanishing average. Plasma losses due to this large-scale electric field are likely to dominate turbulence induced losses caused by plasma instabilities.

We see the possibility of interesting experiments to be carried out in plasma devices like ours $[2,3,4,5]$ : mounting the discharge filament on a radially movable support, it will be possible to study the effects of plasma rotation. The parabolic potential well becomes distorted when moving the filament, and the rotation inhibited when the filament gets close to the wall of the confining vessel. The resulting decrease in plasma confinement should be observable as a decrease in overall plasma density. A related analysis can be carried out by using a segmented limiter. An outer section of this can be moved in the direction of the filament, thereby distorting the steady state potential variation.

\section{Acknowledgments}

The data-sets used in the present analysis were obtained in the Blaamann device at the Auroral Observatory of the University of Troms $\varnothing$. The experiment has now been dismantled on the request of the Norwegian National Science Foundation. We would like to thank colleagues, students, and the technical staff for their contributions to the work on this experiment. In particular, we thank Terje Brundtland for his enthusiasm and tireless work in the construction and maintenance of the device. We thank prof. Odd Erik Garcia for his interest in the problem and for valuable discussions. Bjørn Lybekk kindly offered his assistance with some of the figures. The authors also acknowledge results obtained by Anne Schad Bergsaker during her Master Project. 


\section{Basic features of magnetized plasma torii}

\section{Appendix A. Basic parameter summary}

In Table A1 we give some basic physical parameters for the experiment. The variation of basic parameters as the steady state plasma density $n$, electron temperature $T_{e}$, and plasma potential $\phi_{p}$ are shown in Fig. A1. The electron temperature and plasma potential are found by Langmuir probe characteristics obtained at each spatial position over the entire cross-section with $1.4 \mathrm{~cm}$ separation in the two directions. The plasma density is monitored by the electron saturation current to the Langmuir probe. The results in Fig. A1 refer to time averaged values, while the discussion in Section 3 deals with the fluctuations with respect to these averaged values.

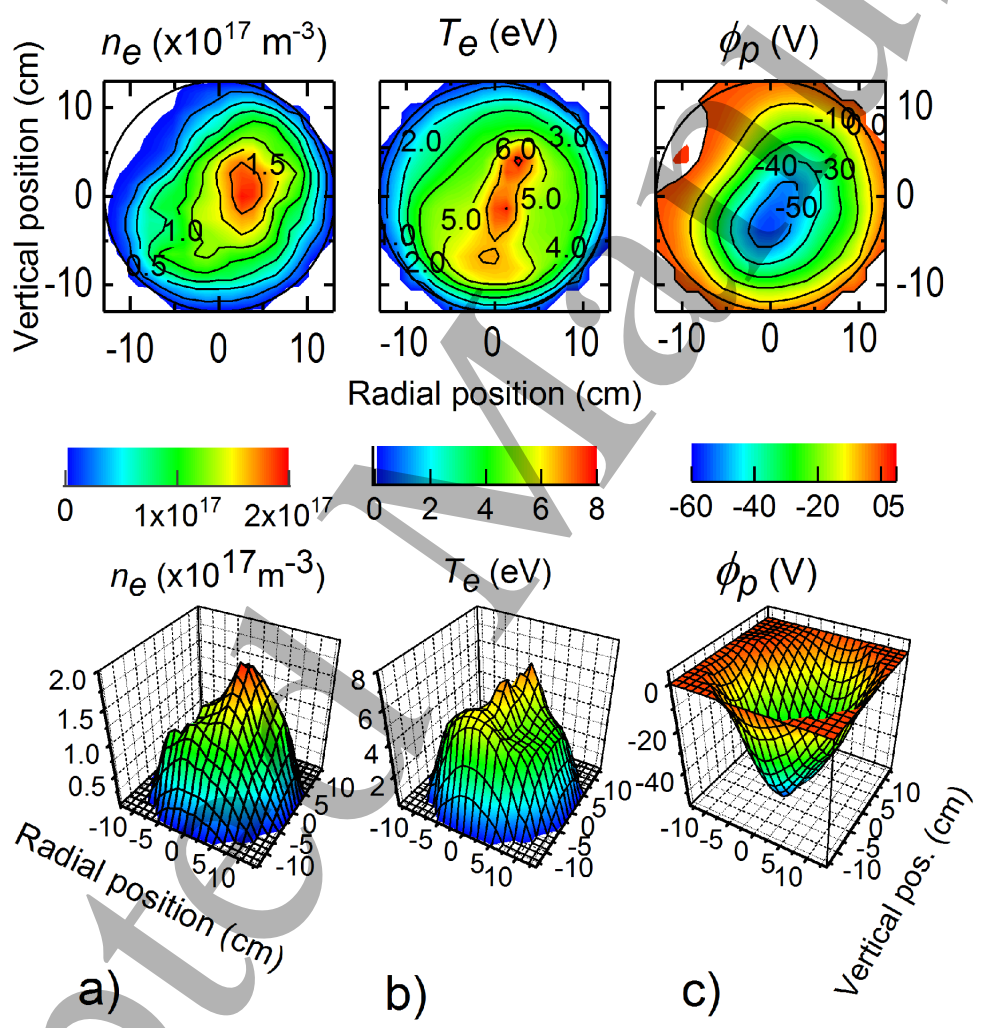

Figure A1. Experimentally obtained variations of the steady state electron density $n_{e}$, électron temperature $T_{e}$, and plasma potential $\phi_{p}$ as measured by Langmuir probes in a cross-section of the plasma torus. The narrow nearly vertical region of elevated electron temperatures indicate the location of the electron emitting discharge filament.

\section{Appendix B. Generalizations of the results for the simple toroidal device}

To the accuracy of the basic model outlined in Section 4, the "top hat" could be solved analytically. The magnetic field inhomogeneity was included through the $\nabla B \times \mathbf{B}$ drift, while we took $\mathbf{B}$ to be a constant elsewhere. For the limit where $\Omega_{p i}^{2} \gg \Omega_{c i}^{2}$ is assumed at all spatial positions, it turns out that the "top hat" restriction is of minor importance as far as the induced polarization electric field is concerned. In the 
1

2

3

4

5

6

7

8

9

10

11

Basic features of magnetized plasma torii

Table A1. Summary of basic plasma parameters, assuming singly charged Helium ions.

\begin{tabular}{|c|c|}
\hline Blaamann major radius $R_{0}$ & $0.67 \mathrm{~m}$ \\
\hline Blaamann minor radius $r_{0}$ & $0.135 \mathrm{~m}$ \\
\hline Toroidal magnetic field at $R_{0}$ & $0.154 \mathrm{~T}$ \\
\hline Vertical magnetic field & $55 \times 10^{-6} \mathrm{~T}$ \\
\hline Neutral He-pressure & $10^{-3}$ mbar \\
\hline Maximum plasma density, $n_{0}$ & $1.6 \times 10^{17}$ \\
\hline Reference electron temperature, $T_{e}$ & $5 \mathrm{eV}$ \\
\hline Ion temperature, $T_{i}$ & 0.05 \\
\hline Electron plasma frequency, $\omega_{p e}$ & $1.8>$ \\
\hline Ion plasma frequency, $\Omega_{p i}$ & $10^{8} \mathrm{~s}^{-1}$ \\
\hline Electron Debye length, $\lambda_{D e}$ & $0^{-6} \mathrm{~m}$ \\
\hline Sound speed, $C_{s}$ & $11 \times 10^{3} \mathrm{~m} \mathrm{~s}^{-1}$ \\
\hline Electron thermal velocity, $u_{t h e}$ & \\
\hline Ion thermal velocity, $u_{t h i}$ & \\
\hline $\begin{array}{l}\text { Electron } \nabla B \times \mathbf{B} \text { and curvature } \\
\text { drift velocity }\end{array}$ & $60 \mathrm{~m} \mathrm{~s}^{-1}$ \\
\hline Ion $\nabla B \times \mathbf{B}$ and curvature drift velocity & $0.6 \mathrm{~m} \mathrm{~s}^{-1}$ \\
\hline Electron cyclotron frequency, $\omega_{c e}$ & $27 \times 10^{9} \mathrm{~s}^{-1}$ \\
\hline Ion cyclotron frequency, $\Omega_{c i}$ & $3.7 \times 10^{6} \mathrm{~s}^{-1}$ \\
\hline Average electron Larmor radius & $35 \times 10^{-6} \mathrm{~m}$ \\
\hline Average ion Larmor radius & $0.27 \times 10^{-3} \mathrm{~m}$ \\
\hline Ion-electron collision frequency, $\nu_{e, i}$ & $80 \times 10^{3} \mathrm{~s}^{-1}$ \\
\hline Electron-neutral He cross-section, $\sigma_{e, n}$ & $6 \times 10^{-20} \mathrm{~m}^{2}$ \\
\hline Ion-neutral He cross-section, $\sigma_{i, n}$ & $65 \times 10^{-20} \mathrm{~m}^{2}$ \\
\hline Electron-neutral mean free path, $\ell_{e, n}$ & $0.7 \mathrm{~m}$ \\
\hline Ion-neutral mean free path, $\ell_{i, n}$ & $64 \times 10^{-3} \mathrm{~m}$ \\
\hline Electron-He collision frequency, $\nu_{e, n}$ & $1.4 \times 10^{6} \mathrm{~s}^{-1}$ \\
\hline Ion-He collision frequency, $\nu_{i, n}$ & $16 \times 10^{3} \mathrm{~s}^{-1}$ \\
\hline$\varepsilon_{r} \triangleq 1+n_{0} M / \varepsilon_{0} B^{2}=1+\left(\Omega_{p i} / \Omega_{c i}\right)^{2}$ & 3100 \\
\hline
\end{tabular}

large plasma density limit we have the relative permittivity to be $\varepsilon_{r} \approx n M / \varepsilon_{0} B^{2}$. The polarization of the plasma is $\mathbf{P}=e n \Delta \mathbf{r}$ with $\Delta r=\left(U_{e}+U_{i}\right) t$ being the relative displacement between electrons and ions due to the $\nabla B \times \mathbf{B}$-drifts. Here we have $\Delta r$ to be linearly increasing with time [19]. The electric field is found by $\nabla \cdot \varepsilon_{0} \varepsilon_{r} \mathbf{E}=-\nabla \cdot \mathbf{P}$, giving $E=e B^{2}\left(U_{e}+U_{i}\right) t / M$. Consequently, we can use a spatially constant polarization electric field $E \approx e B^{2}\left(U_{e}+U_{i}\right) t / M$, which can then be applied for any density profile. This electric field is then added to $E_{0}$ that gives the plasma rotation. The effect of a small vertical magnetic field is not included here since the simple relation between 


\section{Basic features of magnetized plasma torii}

plasma density and electric field will no longer apply in that case. The assumption $\Omega_{p i}^{2} \gg \Omega_{c i}^{2}$ is not fulfilled at the outer edges of the plasma, but we consider this limitation to be of minor consequence here.

The local plasma drift velocity is given now by $\mathbf{U}(\mathbf{r}, t)=\left(\mathbf{E}_{0}(\mathbf{r})+\mathbf{E}(\mathbf{r}, t)\right) \times$ $\mathbf{B}(\mathbf{r}) / B^{2}(\mathbf{r})$. One consequence of this variation is that the flow field $\mathbf{U}(\mathbf{r}, t)$ is no longer incompressible. The rotation velocity $\mathbf{E}_{0}(\mathbf{r}) \times \mathbf{B}(\mathbf{r}) / B^{2}(\mathbf{r})$ is reduced on the high field side and enhanced on the low field side as compared to the case with a constant magnetic field. The plasma density is then slightly larger at the high magnetic field side as compared to the low magnetic field side as caused by the angular variation of the flow velocity in the $x-y$ plane.

Guided by the results from the previous sections we assume that the plasma polarization is at all times vertical in the $y$-direction. The continuity equation for the plasma density becomes

$$
\begin{aligned}
& \frac{\partial n(\mathbf{r}, t)}{\partial t}= \\
& \nabla_{\perp} \cdot\left[\frac{n(\mathbf{r}, t)}{B^{2}}\left(\frac{E_{0}}{r_{0}} \mathbf{r}+\frac{e B^{2}\left(U_{e}+U_{i}\right) t}{M} \widehat{\mathbf{y}}\right) \times \mathbf{B}\right] .
\end{aligned}
$$

Due to the magnetic field dependence of $\varepsilon_{r}$, the electric field induced by the plasma polarization is largest where the magnetic field is largest: we have polarization electric field $\mathbf{E} \sim B^{2}$ implying that the local $\left.\mathbf{E}(\mathbf{r}, t)\right) \times \mathbf{B} / B^{2}$-drift velocity scales linearly with $B$. An initially cylindrically symmetric density distribution will be deformed, becoming elliptical and tilted. The polarization vector remains vertical in the present model, so the potential variations along the $x$-axis will remain in phase, but the density variations will not be so when the distorted density profile rotates due to the $\mathbf{E}_{0}(\mathbf{r}) \times \mathbf{B}(\mathbf{r}) / B^{2}(\mathbf{r})$ rotation.

With $\mathbf{B}=B_{0}\left(1+x / R_{0}\right)^{-1} \widehat{\mathbf{b}}$, the plasma continuity equation becomes

$$
\begin{aligned}
& \frac{\partial n(\mathbf{r}, t)}{\partial t}= \\
& \nabla_{\perp} \cdot\left[n(\mathbf{r}, t)\left(\left(1+\frac{x}{R_{0}}\right) \frac{E_{0}}{B_{0} r_{0}} \mathbf{r}-\frac{e B_{0}\left(U_{e}+U_{i}\right) t}{M\left(1+x / R_{0}\right)} \widehat{\mathbf{y}}\right) \times \widehat{\mathbf{z}}\right] .
\end{aligned}
$$

The first term in the parenthesis of (B.2) originates from the parabolic potential well, the second from the polarization of the plasma by the $\nabla B \times \mathbf{B}$-drift.

The analysis summarized in the present subsection corresponds to the discussion in Section 5.2, i.e. without the effects of vertical magnetic field components, nor ion collisions. Numerical solutions of (B.2) are shown in Fig. B1. The most convenient normalization of positions for the present problem is by the major radius $R_{0}$ of the torus, as seen from (B.2). We find a rotation and a slow average downward drift of the plasma column consistent with features found in Fig. 11. Since (B.2) consistently includes also the effects of the inhomogeneous magnetic field, we find additional features in the form of a deformation of the plasma cross-section together with a concentration of the plasma due to the compressibility of the flow. The calculations based on (B.2) assume high plasma densities, so the results are inaccurate at the outer limits of the 


\section{Basic features of magnetized plasma torii}
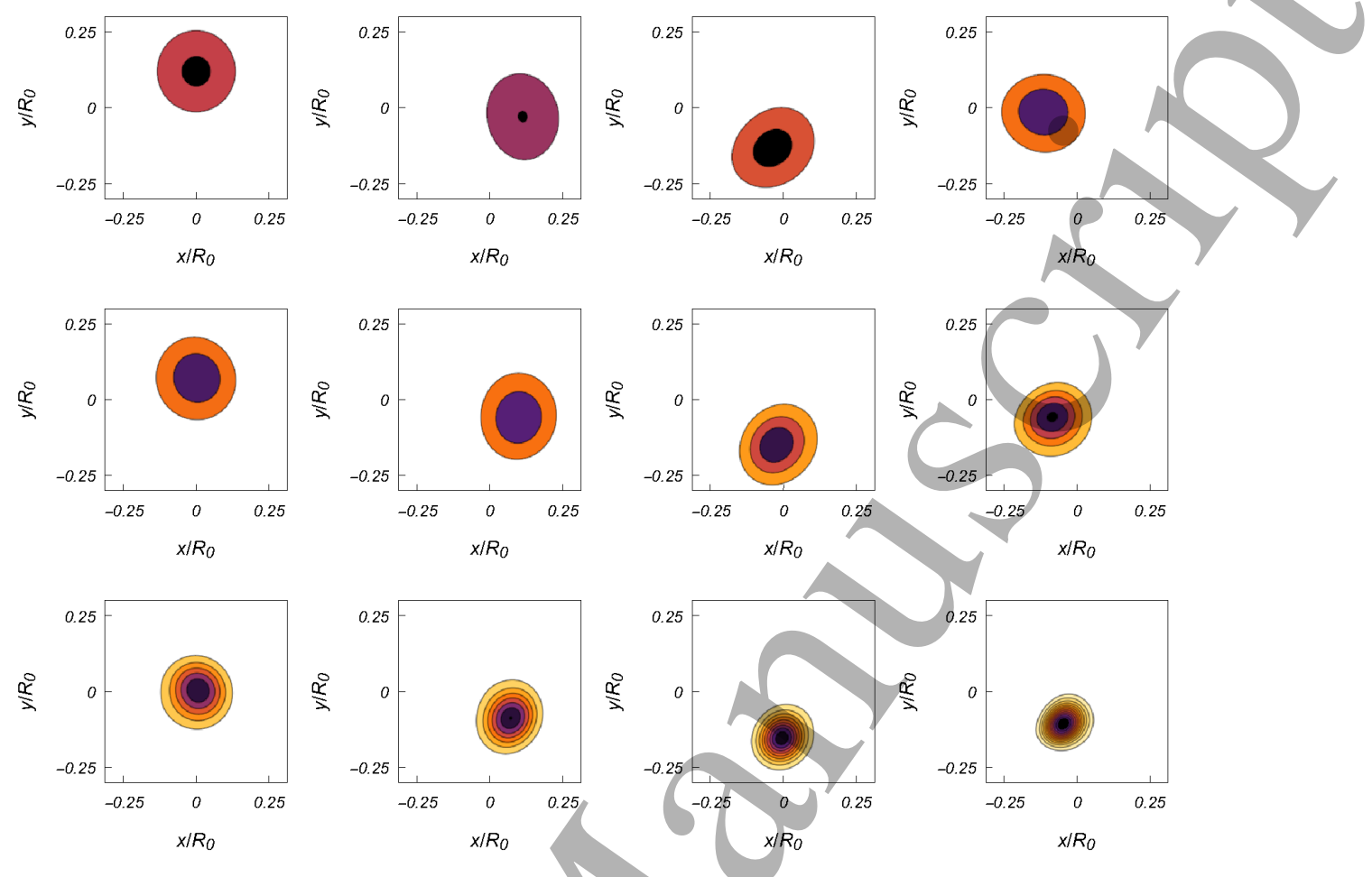

Figure B1. Illustrative numerical solutions of (B.2) for a time interval $t \in\left\{0,6 \pi \Omega_{0}^{-1}\right\}$ with a Gaussian density distribution as initial condition. The time interval between two figures is $\frac{1}{2} \pi \Omega_{0}^{-1}$. Times increase from left to right. Each column corresponds to the same phase in terms of the rotation frequency $\Omega_{0}$. We used $\Omega_{c i, 0}\left(U_{e}+U_{i}\right)\left(\Omega_{0}^{2} R_{0}\right)^{-1}=$ $6 \times 10^{-3}$. Positions are normalized by the major radius $R_{0}$ of the torus. The consequences of the compressible flow are noticeable.

plasma. While it was a relatively simple matter to include the effects of a small vertical magnetic field component as well as ion collisions into the "top hat" model, it is not evident how to modify (B.2) to include similar effects.

\section{References}

[1] K. Rypdal, E. Grønvoll, F. Øynes, Å. Fredriksen, R. J. Armstrong, J. Trulsen, and H. L. Pécseli. Confinement and turbulent transport in a plasma torus with no rotational transform. Plasma Phys. Contr. Fusion, 36:1099-1114, 1994. doi:10.1088/0741-3335/36/7/002.

[2] R. Kaur, A. K. Singh, R. Singh, A. Sarada Sree, and S. K. Mattoo. Territorial characteristics of low frequency electrostatic fluctuations in a simple magnetized torus. Phys. Plasmas, 18:012109, 2011. doi:10.1063/1.3551514.

[3] T. S. Goud, R. Ganesh, Y. C. Saxena, D. Raju, K. Sathyanarayana, K. K. Mohandas, and C. Chavda. Coherent to turbulence transition, enhanced flow and confinement in a simple toroidal plasma. Phys. Plasmas, 19:032307, 2012. doi:10.1063/1.3692227.

[4] U. Kumar, S. G Thatipamula, R. Ganesh, Y. C. Saxena, and D. Raju. Effect of magnetic field topology on quasi-stationary equilibrium, fluctuations, and flows in a simple toroidal device. Phys. Plasmas, 23:102301, 2016. doi:10.1063/1.4964145.

[5] R. Barni, S. Caldirola, L. Fattorini, and C. Riccardi. The different evolution of plasma density voids and blobs in the edge region of a simply magnetized toroidal plasma. Phys. Plasmas, 24:032306, 2017. doi:10.1063/1.4978491.

[6] K. Rypdal, O. E. Garcia, and J.-V. Paulsen. Anomalous cross-field current and 


\section{Basic features of magnetized plasma torii}

fluctuating equilibrium of magnetized plasmas. Phys. Rev. Lett., 79:1857-1860, 1997. doi:10.1103/PhysRevLett.79.1857.

[7] S. Servidio, L. Primavera, V. Carbone, A. Noullez, and K. Rypdal. A model for twodimensional bursty turbulence in magnetized plasmas. Phys. Plasmas, 15:012301, 2008. doi:10.1063/1.2825656.

[8] A. Piel. Plasma Physics: An Introduction to Laboratory, Space, and Fusion Plasmas. Springer, Heidelberg, 2010.

[9] L. Fattorini, Å. Fredriksen, H. L. Pécseli, C. Riccardi, and J. K. Trulsen. Turbulent transport in a toroidal magnetized plasma. Plasma Phys. Control. Fusion, 54:085017, 2012. doi:10.1088/0741$3335 / 54 / 8 / 085017$.

[10] A. S. Bergsaker, Å. Fredriksen, H. L. Pécseli, and J. K. Trulsen. Models for the probability densities of the turbulent plasma flux in magnetized plasmas. Phys. Scripta, 90:108005, 2015. doi:10.1088/0031-8949/90/10/108005.

[11] J. S. Bendat and A. G. Piersol. Random Data, Analysis and Measurement Procedures. John Wiley, New York, 2nd edition, 1986.

[12] N. Aiba, J. Shiraishi, and M. Hirota. Impact of plasma rotation on the linear physics of resistive wall modes in tokamaks. Plasma Phys. Controlled Fusion, 55:074002, 2013. doi:10.1088/0741$3335 / 55 / 7 / 074002$.

[13] F. J. Øynes, O-M. Olsen, H. L. Pécseli, Å. Fredriksen, and K. Rypdal. Experimental study of low-frequency electrostatic fluctuations in a/magnetized toroidal plasma. Phys. Rev. E, 57:22422255, 1998. doi:10.1103/PhysRevE.57.2242.

[14] F. J. Øynes, H. L. Pécseli, and K. Rypdal. Experimental study of low-frequency electrostatic fluctuations in a magnetized toroidal plásma. Phys. Rev. Lett., 75:81-84, 1995. doi:10.1103/PhysRevLett.75.81.

[15] T. Pierre, A. Escarguel, D. Guyomarc'h, R. Barni, and C. Riccardi. Radial convection of plasma structures in a turbulent rotating magnetized-plasma column. Phys. Rev. Lett., 92:065004, 2004. doi:10.1103/PhysRevLett.92.065004.

[16] F. F. Chen. Introduction to Plasma Physics and Controlled Fusion, volume 1. Plenum Press, New York, 2 edition, 1984.

[17] O. E. Garcia. Collective motions in non-uniformly magnetized plasmas. European J. Phys., 24:331-339, 2003. doi:10.1088/0143-0807/24/4/351.

[18] H. L. Pécseli, D. S. Sortland, and O. E. Garcia. A solvable blob-model for magnetized plasmas. Plasma Phys. Controlled Fusion, 58:104002, 2016. doi:10.1088/0741-3335/58/10/104002.

[19] S. Chandrasekhar. Plasma Physics. The University of Chicago Press, Chicago, 1960. Notes compiled by S. K. Trehan after a course given by S. Chandrasekhar.

[20] K. Odajima. Effeets of radial electric-field on drift wave instability in a weakly ionized plasma. J. Phys. Soc. Jap., 44:1685-1693, 1978. doi:10.1143/JPSJ.44.1685.

[21] A. Hasegawa and M. Wakatani. Plasma edge turbulence. Phys. Rev. Lett., 50:682-686, 1983. doi:10.1103/PhysRevLett.50.682.

[22] H. L. Pécseli. Waves and Oscillations in Plasmas. Taylor \& Francis, London, 2012.

[23] T. Mikkelsen and H. L. Pécseli. Strong turbulence in partially ionized plasmas. Phys. Lett. A, 77:159-162, 1980. doi:10.1016/0375-9601(80)90179-6.

[24] D. Bora. Curvature induced low frequency instabilities in a toroidal plasma. Phys. Lett. A, 139:308-312, 1989. doi:10.1016/0375-9601(89)90458-1. 